\title{
A Review on the Terpenes from Genus Vitex
}

\author{
Jin-Long Yao ${ }^{1,2, \dagger}{ }^{\dagger}$, Shi-Ming Fang ${ }^{1,2,+}$, Rui Liu ${ }^{1,3}$, Mahmood Brobbey Oppong ${ }^{1}$, Er-Wei Liu ${ }^{1,2}$, \\ Guan-Wei Fan ${ }^{1,2, *}$ and Han Zhang ${ }^{1,2, *}$ \\ 1 Tianjin State Key Laboratory of Modern Chinese Medicine, Tianjin University of Traditional Chinese \\ Medicine, 312 Anshanxi Road, Nankai District, Tianjin 300193, China; yaojinlong1992@163.com (J.-L.Y.); \\ fang_shiming@163.com (S.-M.F.); 1r_8000@163.com (R.L.); mahmood.obrobbey@gmail.com (M.B.O.); \\ liuwei628@sohu.com (E.-W.L.) \\ 2 Key Laboratory of Formula of Traditional Chinese Medicine of Ministry of Education, \\ Tianjin University of Traditional Chinese Medicine, 312 Anshanxi Road, Nankai District, \\ Tianjin 300193, China \\ 3 School of Chinese Materia Medica, Tianjin University of Traditional Chinese Medicine, 312 Anshanxi Road, \\ Nankai District, Tianjin 300193, China \\ * Correspondence: fgw1005@163.com (G.-W.F.); zhanghan0023@126.com (H.Z.); \\ Tel./Fax: +86-22-5959-6163 (G.-W.F.); +86-22-5959-6176 (H.Z.) \\ + These authors contributed equally to this work. \\ Academic Editor: Derek J. McPhee \\ Received: 19 July 2016; Accepted: 1 September 2016; Published: 6 September 2016
}

\begin{abstract}
The genus Vitex, which belongs to the Verbenaceae family, includes approximately 250 species. Some species of the genus Vitex have traditionally been used for the treatment of headaches, ophthalmodynia, coughs, asthma, premenopausal syndrome, etc. Chemical investigations indicate that the characteristic constituents of the genus Vitex are terpenes, and 210 of these compounds, including monoterpenoids, sesquiterpenoids, diterpenoids and triterpenoids, have been obtained from 12 species. Pharmacological studies had shown that these terpenes possess anti-inflammatory, antitumor, antibacterial, antioxidant activities, and so on. In this paper, the identity of these terpenes and their pharmacological effects are reviewed, which can provide references for further research regarding the chemistry and utilization of the Vitex species.
\end{abstract}

Keywords: Vitex genus; chemical constituents; terpenes; pharmacological effects

\section{Introduction}

The genus Vitex is one of the largest genus in the Verbenaceae family, with approximately 250 species. It is widely distributed, but mainly found in the tropical areas with a few in subtropical regions. The plants are mostly shrubs or arbors [1]. Many species in the Vitex genus have significant medicinal effects. The fruits of Vitex trifolia L. var. simplicifolia Cham. and Vitex trifolia L. are named Manjingzi in the 2015 edition of Chinese Pharmacopoeia. Manjingzi is a traditional Chinese medicine with wind-heat-dispersing action used in treating headaches, migraines and ophthalmodynia. The leaves of V. negundo var. cannabifolia have been used in China for the treatment of coughs, phlegm, and asthma [2]. Various parts of V. negundo, including the leaves, roots and seeds, have been locally used as traditional folk medicines since antiquity, particularly in China. It is commonly used for its analgesic, anti-inflammatory, anti-rheumatism, and insecticidal effects [3]. Many other species of the genus also have been explored and researched. These include $V$. agnus-castus, V. limonifolia, $V$. altissima, V. rotundifolia, V. peduncularis, V. negundo var. cannabifolia, $V$. vestita, V. rehmannii, etc.

Different types of secondary metabolites e.g., terpenes, flavonoids, lignans, phenolic acids, anthraquinones, etc., are present in species in this genus [4]. Terpenes are one of the major secondary metabolites, with different types including monoterpenoids, sesquiterpenoids, diterpenoids, 
and triterpenoids being isolated and characterized from the genus. Pharmacological studies have shown that terpenes have anti-inflammatory, antitumor, antibacterial, antioxidant, hepatoprotective activities and so on. The goal of this review is to provide an overview of the chemical identities and the pharmacological effects of the terpenes isolated from species in the genus, which can serve as reference for further research and utilization of the Vitex species.

\section{Chemical Constituents}

So far, more than 200 terpenes have been obtained from the different parts of Vitex plants. Among these compounds, diterpenoids are the most dominant terpenes reported in the species. The names of terpenes, the corresponding plant sources and references from which they are derived are summarized in Tables 1-4. Their structures are shown in Figures 1-4.

\subsection{Monoterpenoids and Sesquiterpenoids}

\subsubsection{Monoterpenoids}

The majority of the monoterpenoids (Table 1, Figure 1) of the Vitex genus are iridoids and their corresponding glucosides (compounds 1-31). Beside the iridoids, two cineole-type monoterpenoid glucosides 32, 33 were obtained from the fruits of Vitex rotundifolia [5]. Moreover, Wu et al. [6] isolated an acyclic monoterpenoid vitexoid 34 from the fruits of Vitex trifolia.

Table 1. Monoterpenoids 1-34 isolated from plants of Vitex L.

\begin{tabular}{|c|c|c|c|}
\hline No. & Compound Name & Source & Reference \\
\hline 1 & Nishindaside & $\mathrm{a}, \mathrm{h}$ & [7-9] \\
\hline 2 & Isonishindaside & $\mathrm{h}$ & {$[8]$} \\
\hline 3 & Agnuside & $a-h$ & [9-21] \\
\hline 4 & 10-O-Vanilloyl aucubin & $\mathrm{d}, \mathrm{e}, \mathrm{h}$ & {$[9,14-16]$} \\
\hline 5 & Limoniside & $\mathrm{g}$ & [10] \\
\hline 6 & Aucubin & $\mathrm{f}$ & {$[11,18]$} \\
\hline 7 & Eurostoside & d & [12] \\
\hline 8 & Harpagide & $\mathrm{f}$ & [22] \\
\hline 9 & 8-O-Acetylharpagide & $\mathrm{f}$ & [22] \\
\hline 10 & Geniposide & $\mathrm{h}$ & [9] \\
\hline 11 & Mussaenosidic acid & $\mathrm{f}$ & [11] \\
\hline 12 & $6^{\prime}-O-p$-Hydroxybenzoylmussaenosidic acid & $a, b, f$ & {$[11,21,23]$} \\
\hline 13 & Agnucastoside A & $\mathrm{f}$ & [11] \\
\hline 14 & Agnucastoside B & $\mathrm{f}$ & [11] \\
\hline 15 & Agnucastoside C & $\mathrm{f}$ & [11] \\
\hline 16 & $2^{\prime}$-O-trans-p-Coumaroylloganic acid & a & [23] \\
\hline 17 & $6^{\prime}$-O-trans-Feruloylnegundoside & $\mathrm{c}$ & [13] \\
\hline 18 & $6^{\prime}$-O-trans-Caffeoylnegundoside & c & [13] \\
\hline 19 & $2^{\prime}$-O- $p$-Hydroxybenzoyl-6'-O-trans-caffeoylgardoside & $\mathrm{c}$ & [13] \\
\hline 20 & $2^{\prime}$-O-p-Hydroxybenzoyl-6'-O-trans-caffeoyl-8-epiloganic acid & c & [13] \\
\hline 21 & $2^{\prime}-\mathrm{O}-p$-Hydroxybenzoyl gardoside & c & [13] \\
\hline 22 & $2^{\prime}-O-p$-Hydroxybenzoyl-8-epiloganic acid & c & [13] \\
\hline 23 & Negundoside & a, c & {$[7,13,23-27]$} \\
\hline 24 & $6^{\prime}$-O-p-Hydroxybenzoyl-gardoside & a & [23] \\
\hline 25 & $\begin{array}{c}\text { 1,4a,5,7a-Tetrahydro-1- } \beta \text {-D-glucosyl-7- }\left(3^{\prime}, 4^{\prime}-\right. \\
\text { dihydroxybenzoyloxymethyl)-5-ketocyclopenta[c]pyran-4-carboxylic acid }\end{array}$ & a & [7] \\
\hline 26 & Iridolactone & $\mathrm{d}$ & [14] \\
\hline 27 & Viteoid II & $\mathrm{d}$ & [14] \\
\hline 28 & Viteoid I & $\mathrm{d}$ & [14] \\
\hline 29 & Pedicularis lactone & $\mathrm{d}$ & [14] \\
\hline 30 & Eucommiol & $\mathrm{d}$ & [14] \\
\hline 31 & 1-Oxoeucommiol & $\mathrm{d}$ & [14] \\
\hline 32 & $(1 S, 2 S, 4 R)$-2-endo-Hydroxy-1,8-cineole- $\beta$-D-glucopyranoside & $\mathrm{d}$ & [5] \\
\hline 33 & $(1 R, 2 R, 4 S)$-2-endo-Hydroxy-1,8-cineole- $\beta$-D-glucopyranoside & $\mathrm{d}$ & [5] \\
\hline 34 & Vitexoid & $\mathrm{b}$ & [6] \\
\hline
\end{tabular}

a: Vitex negundo. b: V. trifolia. c: V. altissima. d: V. rotundifolia. e: V. peduncularis. f: V. agnus-castus. g: V. limonifolia. h: V. negundo var. cannabifolia (syn.: V. cannabifolia). 


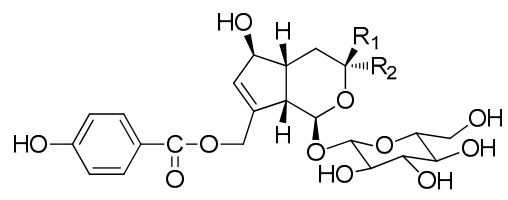

$1 \mathrm{R}_{1}=\mathrm{OCH}_{3} \mathrm{R}_{2}=\mathrm{H}$

$2 \mathrm{R}_{1}=\mathrm{H} \mathrm{R}_{2}=\mathrm{OCH}_{3}$

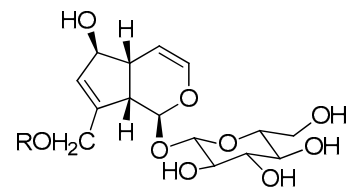

7 R=trans-p-hydroxycinnamoyl

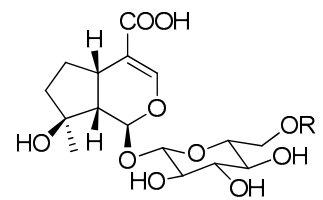

$11 \mathrm{R}=\mathrm{H}$

$12 \mathrm{R}=p-\mathrm{OH}-\mathrm{Ph}-\mathrm{CO}$

$13 \mathrm{R}=$ foliamenthoyl

$14 \mathrm{R}=6,7$-dihydrofoliamenthoyl<smiles>[R]C1C[C@H]2C(C(=O)O)=CO[C@H](OCC)[C@@]2([R5])C1([R])[R]</smiles>
$\mathrm{COOH}$

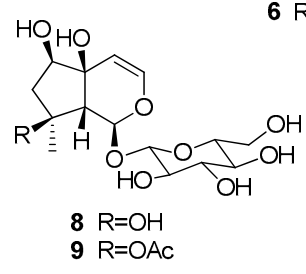

$4 \mathrm{R}=p-\mathrm{OH}-m-\mathrm{OCH}_{3}-\mathrm{Ph}-\mathrm{CO}$

$\mathrm{R}=p-\mathrm{OCH}_{3}-\mathrm{Ph}-\mathrm{CO}-$

$\mathrm{R}=\mathrm{H}$<smiles>COCC(=O)OC</smiles>

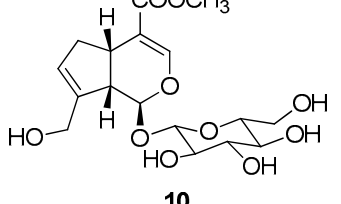

10

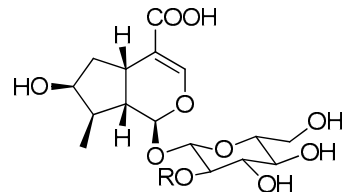

$16 \mathrm{R}=$ trans $-p$ coumaroyl
$15 \mathrm{R}_{1}=$ trans- $p$-coumaroyl $\mathrm{R}_{2}=$ trans-caffeoyl $17 \begin{array}{llll}\mathrm{R}_{1} & \mathrm{R}_{2} & \mathrm{R}_{3} & \mathrm{R}_{4}\end{array}$ $\begin{array}{llll} & \mathrm{OH} & \mathrm{CH}_{3} & \text { trans-feruloyl }\end{array}$ $18 \mathrm{H} \quad \mathrm{OH} \quad \mathrm{CH}_{3}$ trans-caffeoyl $19 \mathrm{OH} \quad=\mathrm{CH}_{2}$ trans-caffeoyl $20 \mathrm{OH} \quad \mathrm{H} \quad \mathrm{CH}_{3}$ trans-caffeoyl $21 \mathrm{OH}$ $22 \mathrm{OH}$ $\begin{array}{lll}=\mathrm{CH}_{2} & \mathrm{H} \\ \mathrm{CH}_{3} & \mathrm{H}\end{array}$

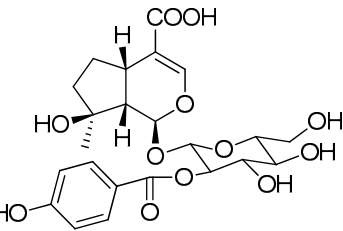<smiles>[R]OC1CCC1([R])O</smiles>

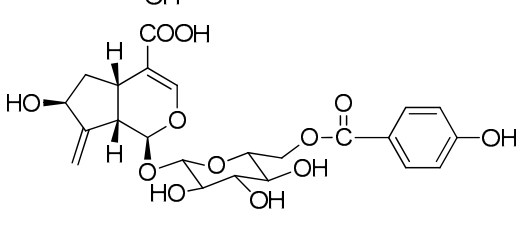<smiles>O=C(O)C1=COC(OC2OC(O)C(O)C(O)C(O)C2O)C23C(COC(=O)c4ccc(O)c(O)c4)C=CC(=O)C12C3O</smiles>

24<smiles>O=C1C[C@H]2C=C(CO)C(=O)C[C@H]2O1</smiles>

29<smiles>O=C1C[C@H]2C=C(CO)C(=O)C[C@]2(CO)CO1</smiles><smiles>O=C1OCC[C@@H]2C1=C(CO)CC2O</smiles><smiles>O=C1OCC2=C1[C@H](CCO)C(O)C2</smiles>

28<smiles>CC1(C)OC2CCC1(C)OC2OC(O)C(O)CO</smiles>

33<smiles>[R]C(O)C1=C(CO)CC(O)C1CCO</smiles>

$30 \mathrm{R}=\mathrm{H}_{2}$<smiles>CC12CCC(C)(OC1OC(O)C1OC(O)C(O)C1O)C(C)(C)O2</smiles>

Figure 1. Structures of monoterpenoids 1-34 isolated from plants of Vitex L.

\subsubsection{Sesquiterpenoids}

Apart from the monoterpenoids, only eight sesquiterpenoids 35-42 were found in the Vitex plants (Table 2, Figure 2). Among them, negunfurol (35) is a new sesquiterpenoid from $V$. negundo containing a furan ring [28]. Tiwari et al. [29] isolated three sesquiterpenoids 36-38 with furanoeremophilane 
skeletons from the stems of $V$. negundo. Meanwhile, aromadendrane-type sesquiterpenoids 39-42 have been obtained from $V$. trifolia, V. agnus-castus and V. poligama [17,18,30-33].

Table 2. Sesquiterpenoids 35-42 isolated from plants of Vitex L.

\begin{tabular}{cccc}
\hline No. & Compound Name & Source & Reference \\
\hline $\mathbf{3 5}$ & Negunfurol & $\mathrm{a}$ & {$[28]$} \\
$\mathbf{3 6}$ & 1,6-Dioxo-2(3),9(10)-dehydrofuranoeremophilane & $\mathrm{a}$ & {$[29]$} \\
$\mathbf{3 7}$ & 4,6-Dimethyl-11-formyl-1-oxo-4H,2,3-dihydronaphthofuran & $\mathrm{a}$ & {$[28,29]$} \\
$\mathbf{3 8}$ & $4,6-$ Dimethyl-11-dimethoxymethyl-1-oxo-4H,2,3-dihydronaphthofuran & $\mathrm{a}$ & {$[29]$} \\
$\mathbf{3 9}$ & Spathulenol & $\mathrm{b}, \mathrm{f}, \mathrm{i}$ & {$[17,18,30,31]$} \\
$\mathbf{4 0}$ & ent-4 $\alpha, 10 \beta$-Dihydroxyaromadendrane & $\mathrm{b}$ & {$[17]$} \\
$\mathbf{4 1}$ & $4 \beta, 10 \beta$-Dihydroxyaromadendrane & $\mathrm{f}$ & {$[32]$} \\
$\mathbf{4 2}$ & $4 \alpha, 10 \alpha$-Dihydroxyaromadendrane & $\mathrm{f}$ & {$[33]$} \\
\hline
\end{tabular}

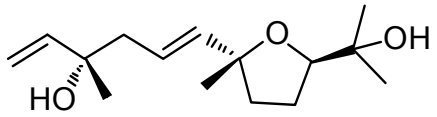

35<smiles>Cc1coc2c1C(=O)[C@@]1(C)C(=C2)C(=O)C=C[C@H]1C</smiles>

36<smiles>[R]c1coc2cc3c(c(C)c12)[C@@H](C)CCC3=O</smiles>

$37 \mathrm{R}=\mathrm{CHO}$

$38 \mathrm{R}=\mathrm{CH}\left(\mathrm{OCH}_{3}\right)_{2}$

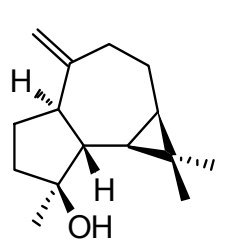

39

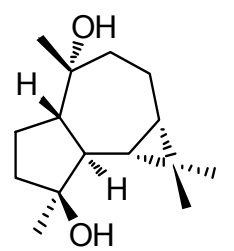

40

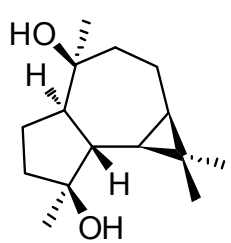

41

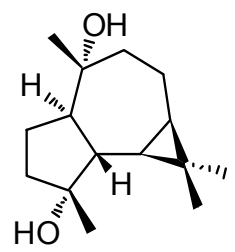

42

Figure 2. Structures of sesquiterpenoids 35-42 isolated from plants of Vitex L.

\subsection{Diterpenoids}

Diterpenoids are abundant in the Vitex plants. The labdane-type diterpenoids 43-120 form the majority of the characterized diterpenoids, with the few others being norlabdane-type (compounds 121-132), halimane-type (compounds 133-141), abietane-type (compounds 142-151), clerodane-type (compounds 152-155) and isopimarane-type (compound 156). Commonly, diterpenoids of the genus exist in the form of aglycones, and only 65 and 66 are diterpenoid glucosides which are rare in Vitex genus [33,34]. Compounds 73, 74 and 75 are found as diterpenoid alkaloids containing an $\alpha, \beta$-unsaturated- $\gamma$-lactam moiety, and these structures are unique in the genus $[18,35,36]$. Zheng et al. [37] isolated a 9,10-seco abietane diterpenoid negundoin F (151) and an isopimarane-type diterpenoid negundoin G (156) from an ethanolic extract of the seeds of $V$. negundo. The names of diterpenoids and their structures are listed and shown in Table 3 and Figure 3, respectively. 
Table 3. Diterpenoids 43-156 isolated from plants of Vitex L.

\begin{tabular}{|c|c|c|c|c|}
\hline Type & No. & Compound Name & Source & Reference \\
\hline \multirow{38}{*}{ Labdane } & 43 & Rotundifuran & $b, d, f$ & {$[31,35,38-42]$} \\
\hline & 44 & Vitetrifolin B & $\mathrm{b}, \mathrm{f}$ & {$[31,39]$} \\
\hline & 45 & Dihydrosolidagenone & $\mathrm{b}$ & [39] \\
\hline & 46 & (+)-Polyalthic acid & a & [43] \\
\hline & 47 & Vitetrifolin C & $b, f$ & {$[31,39]$} \\
\hline & 48 & Vitetrifolin $\mathrm{H}$ & $\mathrm{b}$ & {$[6,38]$} \\
\hline & 49 & Vitexilactone C & $\mathrm{h}$ & [44] \\
\hline & 50 & Vitextrifolin C & $\mathrm{b}$ & [38] \\
\hline & 51 & Vitextrifolin D & $\mathrm{b}$ & [38] \\
\hline & 52 & Vitextrifolin E & $\mathrm{b}$ & [38] \\
\hline & 53 & Vitexilactone & $b, d, f$ & {$[6,31,33,35,36,40,45-50]$} \\
\hline & 54 & (rel 5S,6R,8R,9R,10S)-6-Acetoxy-9-hydroxy-13(14)-labden-16,15-olide & $\mathrm{b}, \mathrm{d}$ & {$[6,48,50]$} \\
\hline & 55 & (rel 5S,6S,8R,9R,10S)-6-Acetoxy-9-hydroxy-13(14)-labden-16,15-olide & $\mathrm{d}$ & [50] \\
\hline & 56 & (rel 5S,6R,8R,9R,10S)-6-Acetoxy-9-hydroxy-15-methoxy-13(14)-labden-16,15-olide & d & {$[46,50]$} \\
\hline & 57 & Viteagnusin I & $b, d, f$ & {$[38,45,51]$} \\
\hline & 58 & Viteagnusin $\mathrm{H}$ (methoxy-vitexilactone) & $\mathrm{f}$ & {$[32,52]$} \\
\hline & 59 & 9-Hydroxy-13(14)-labden-15,16-olide & $\mathrm{b}$ & [53] \\
\hline & 60 & Deacetylvitexilactone & $\mathrm{b}$ & [38] \\
\hline & 61 & Viterotulin A & $\mathrm{d}$ & [45] \\
\hline & 62 & (rel 3S,5S,8R,9R,10S)-3,9-Dihydroxy-13(14)-labden-16,15-olide & $\mathrm{d}$ & [45] \\
\hline & 63 & Viterotulin B & $\mathrm{d}$ & [45] \\
\hline & 64 & Vitexilactone B & $\mathrm{a}, \mathrm{b}$ & {$[38,54]$} \\
\hline & 65 & Viteoside A & $\mathrm{d}$ & [34] \\
\hline & 66 & Viteagnuside A & $\mathrm{f}$ & [33] \\
\hline & 67 & Vitexolide E & $\mathrm{j}$ & {$[55]$} \\
\hline & 68 & Vitexolide D & $\mathrm{j}$ & [55] \\
\hline & 69 & Vitexolide A & $\mathrm{j}$ & [55] \\
\hline & 70 & 12-Epivitexolide A & j & [55] \\
\hline & 71 & Vitexolide B & $\mathrm{j}$ & {$[55]$} \\
\hline & 72 & Vitexolide C & j & {$[55]$} \\
\hline & 73 & Vitexlactam A & $d, f$ & {$[18,36]$} \\
\hline & 74 & Vitexlactam B & $\mathrm{f}$ & {$[18,35]$} \\
\hline & 75 & Vitexlactam C & $\mathrm{f}$ & {$[18,35]$} \\
\hline & 76 & 12S,16S/R-Dihydroxy-ent-labda-7,13-dien-15,16-olide & $\mathrm{k}$ & [56] \\
\hline & 77 & Vitextrifolin G & $\mathrm{b}$ & [38] \\
\hline & 78 & Prerotundifuran & $\mathrm{d}$ & [42] \\
\hline & 79 & Previtexilactone & $\mathrm{b}, \mathrm{d}$ & {$[6,38,47-49]$} \\
\hline & 80 & 6-Acetoxy-9,13,15,16-diepoxy-15-methoxylabdane & $\mathrm{b}$ & [6] \\
\hline
\end{tabular}


Table 3. Cont.

\begin{tabular}{|c|c|c|c|c|}
\hline Type & No. & Compound Name & Source & Reference \\
\hline \multirow{40}{*}{ Labdane } & 81 & Viteagnusin E & $\mathrm{f}$ & {$[57]$} \\
\hline & 82 & (rel 5S,6R,8R,9R,10S,13S)-6-Acetoxy-9,13-epoxy-15-methoxylabdan-16,15-olide & $d, f$ & {$[33,50]$} \\
\hline & 83 & Viteagnusin I & $\mathrm{f}$ & [33] \\
\hline & 84 & (rel 5S,6R,8R,9R,10S,13S,16S)-6-Acetoxy-9,13-epoxy-16-methoxy-labdan-15,16-olide & $d, f$ & {$[33,50]$} \\
\hline & 85 & Vitextrifolin F & $\mathrm{b}$ & [38] \\
\hline & 86 & Nishindanol & a & [19] \\
\hline & 87 & (rel 5S,6R,8R,9R,10S,13S,15S)-6-Acetoxy-9,13,15,16-diepoxy-15-methoxylabdane & $d, f$ & {$[32,58]$} \\
\hline & 88 & (rel $5 S, 6 R, 8 R, 9 R, 10 S, 13 S, 15 R)$-6-Acetoxy-9,13;15,16-diepoxy-15-methoxylabdane & $\mathrm{d}, \mathrm{f}$ & {$[32,58]$} \\
\hline & 89 & (rel $5 S, 6 R, 8 R, 9 R, 10 S, 13 S, 15 S, 16 R)$-6-Acetoxy-9,13,15,16-diepoxy-15,16-dimethoxylabdane & $\mathrm{d}$ & {$[58]$} \\
\hline & 90 & (rel $5 S, 6 R, 8 R, 9 R, 10 S, 13 S, 15 R, 16 S)$-6-Acetoxy-9,13,15,16-diepoxy-15,16-dimethoxylabdane & $\mathrm{d}$ & [58] \\
\hline & 91 & (rel $5 S, 6 R, 8 R, 9 R, 10 S, 13 S, 15 R, 16 R)$-6-Acetoxy-9,13,15,16-diepoxy-15,16-dimethoxylabdane & $\mathrm{d}$ & [58] \\
\hline & 92 & (rel $5 S, 6 R, 8 R, 9 R, 10 S, 13 S, 15 S, 16 S)$-6-Acetoxy-9,13,15,16-diepoxy-15,16-dimethoxylabdane & $\mathrm{d}$ & [58] \\
\hline & 93 & $($ rel $5 S, 8 R, 9 R, 10 S, 13 S, 15 S, 16 R)-9,13 ; 15,16$-Diepoxy-15,16-dimethoxylabdane & $\mathrm{d}$ & [50] \\
\hline & 94 & (rel $5 S, 8 R, 9 R, 10 S, 13 S, 15 R, 16 S)-9,13 ; 15,16$-Diepoxy-15,16-dimethoxylabdane & $\mathrm{d}$ & [50] \\
\hline & 95 & (rel 5S,8R,9R,10S,13S,15R,16R)-9,13;15,16-Diepoxy-15,16-dimethoxylabdane & $\mathrm{d}$ & [50] \\
\hline & 96 & Negundol & $\mathrm{a}, \mathrm{b}$ & {$[38,59]$} \\
\hline & 97 & Negundoin D & $\mathrm{a}$ & [37] \\
\hline & 98 & Negundoin E & a & [37] \\
\hline & 99 & Vitextrifolin A & $\mathrm{b}$ & [38] \\
\hline & 100 & Vitextrifolin B & $\mathrm{b}$ & [38] \\
\hline & 101 & (rel 5S,6R,8R,9R,10S,13R)-6-Acetoxy-9,13-epoxy-15-methoxylabdan-16,15-olide & $\mathrm{d}, \mathrm{f}$ & {$[33,50,57]$} \\
\hline & 102 & (rel 5S,6R,8R,9R,10S,13R,16S)-6-Acetoxy-9,13-epoxy-16-methoxylabdan-15,16-olide & $\mathrm{d}, \mathrm{f}$ & {$[33,50]$} \\
\hline & 103 & Viteagnusin J & $\mathrm{f}$ & [33] \\
\hline & 104 & (rel $5 S, 6 R, 8 R, 9 R, 10 S, 13 R, 15 R)$-6-Acetoxy-9,13,15,16-diepoxy-15-methoxylabdane & $d, f$ & {$[32,58]$} \\
\hline & 105 & (rel 5S,6R,8R,9R,10S,13R,15S)-6-Acetoxy-9,13,15,16-diepoxy-15-methoxylabdane & $\mathrm{d}, \mathrm{f}$ & {$[32,58]$} \\
\hline & 106 & Viteagnusin F & $\mathrm{d}, \mathrm{f}$ & {$[32,45]$} \\
\hline & 107 & Viteagnusin G & $d, f$ & {$[32,45]$} \\
\hline & 108 & Limonidilactone & $\mathrm{g}$ & {$[60]$} \\
\hline & 109 & Acuminolide & $\stackrel{\circ}{j}$ & [55] \\
\hline & 110 & Vitexolin A & $\mathrm{j}$ & [55] \\
\hline & 111 & Vitexolin B & i & [55] \\
\hline & 112 & $6 \alpha, 7 \alpha$-Diacetoxy-13-hydroxy-8(9),14-labdadiene & $\mathrm{b}$ & [53] \\
\hline & 113 & $6 \beta, 7 \beta$-Diacetoxy-13-hydroxylabda-8,14-diene & $\mathrm{f}$ & {$[40,41]$} \\
\hline & 114 & Viteagnusin D & $\mathrm{f}$ & [57] \\
\hline & 115 & Vitexifolin A & d & [61] \\
\hline & 116 & Viteagnusin C & $\mathrm{a}, \mathrm{f}$ & {$[33,54,57]$} \\
\hline & 117 & 8,13-Dihydroxy-14-labdene & $\mathrm{f}$ & [41] \\
\hline & 118 & 8-epi-Sclareol & $\mathrm{a}, \mathrm{f}$ & {$[19,33,54,57]$} \\
\hline & 119 & Vitrifolin B & $\mathrm{d}$ & [36] \\
\hline & 120 & 8-Epimanoyl oxide & $\mathrm{f}$ & {$[18,35,51]$} \\
\hline
\end{tabular}


Table 3. Cont.

\begin{tabular}{|c|c|c|c|c|}
\hline Type & No. & Compound Name & Source & Reference \\
\hline \multirow{12}{*}{ Norlabdane } & 121 & Vitrifolin A & 1 & [62] \\
\hline & 122 & Negundoal & a & [28] \\
\hline & 123 & Negundoin A & a & [37] \\
\hline & 124 & Negundoin B & a & [37] \\
\hline & 125 & Negundoin C & a & [37] \\
\hline & 126 & 9,13-Epoxy-16-norlabda-13E-en-15-al (norditerpene aldehyde 1) & $b, d$ & {$[45,48]$} \\
\hline & 127 & Norditerpene aldehyde 2 & $\mathrm{~b}$ & [48] \\
\hline & 128 & Vitexifolin D & $\mathrm{d}$ & [61] \\
\hline & 129 & Trisnor- $\gamma$-lactone & $\mathrm{d}$ & [61] \\
\hline & 130 & Isoambreinolide & $b, d$ & {$[53,61]$} \\
\hline & 131 & Vitedoin B & $\mathrm{a}, \mathrm{d}$ & {$[37,45,63]$} \\
\hline & 132 & Vitexifolin E & $\mathrm{d}$ & [61] \\
\hline \multirow{9}{*}{ Halimane } & 133 & Vitetrifolin G & $\mathrm{b}$ & [64] \\
\hline & 134 & 13-Hydroxy-5(10),14-halimadien-6-one & $\mathrm{b}$ & [53] \\
\hline & 135 & Viteagnusin A & $\mathrm{f}$ & [57] \\
\hline & 136 & Viteagnusin B & $\mathrm{f}$ & [57] \\
\hline & 137 & Vitetrifolin I & $\mathrm{b}$ & [6] \\
\hline & 138 & Vitetrifolin D & $a, b, d, f$ & {$[6,19,33,35,45,46,52,54,61,64]$} \\
\hline & 139 & Vitetrifolin E & $\mathrm{b}, \mathrm{d}$ & {$[6,45,46,64]$} \\
\hline & 140 & Vitetrifolin F & $\mathrm{b}, \mathrm{d}$ & {$[6,45,46,64]$} \\
\hline & 141 & Vitetrifolin $\mathrm{H}$ & $\mathrm{d}$ & [45] \\
\hline \multirow{10}{*}{ Abietane } & 142 & Ferruginol & $\mathrm{d}$ & {$[58]$} \\
\hline & 143 & 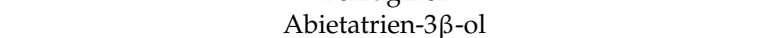 & $b, d$ & {$[39,58]$} \\
\hline & 144 & $5 \beta$-Hydro-8,11,13-abietatrien- $6 \alpha$-ol & a & [65] \\
\hline & 145 & $3 \beta$-Hydroxyabieta-8,11,13-trien-7-one & a & [37] \\
\hline & 146 & Isolophanthin A & d & [45] \\
\hline & 147 & Abieta-9(11),12-diene & $\mathrm{d}$ & [66] \\
\hline & 148 & Vitetrifolin A & $\mathrm{b}$ & [39] \\
\hline & 149 & Abietane 9(11):12(13)-di- $\alpha$-epoxide & d & [66] \\
\hline & 150 & Vitexifolin C & $\mathrm{d}$ & [61] \\
\hline & 151 & Negundoin F & $\mathrm{a}$ & [37] \\
\hline \multirow{4}{*}{ Clerodane } & 152 & Vitexifolin B & $\mathrm{d}$ & [61] \\
\hline & 153 & Cleroda-7,14-dien-13-ol & $\mathrm{f}$ & [41] \\
\hline & 154 & Cleroda-1,3,14-trien-13-ol & $\mathrm{f}$ & [41] \\
\hline & 155 & 13-epi-2-Oxokolavelool & d & [45] \\
\hline Isopimarane & 156 & Negundoin G & a & [37] \\
\hline
\end{tabular}

a: Vitex negundo. b: V. trifolia. d: V. rotundifolia. f: V. agnus-castus. g: V. limonifolia. h: V. negundo var. cannabifolia (syn.: V. cannabifolia). j: V. vestita. k: V. rehmannii. 1: V. trifolia L var. simplicifolia. 


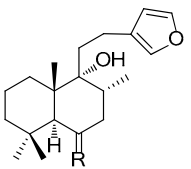

$43 \mathrm{R}=\alpha-\mathrm{H}, \beta-\mathrm{OAC}$ $44 \mathrm{R}=\alpha-\mathrm{O} A \mathrm{c}, \beta-\mathrm{H}$ $45 \mathrm{R}=\mathrm{O}$

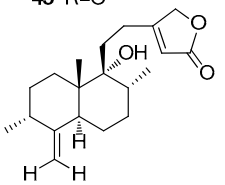

49

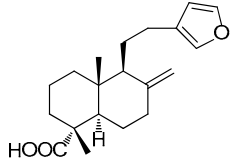

46<smiles>CC(=O)OC1CC(C)=C(CCC2=C(C)C(=O)OC2)[C@]2(C)CCCC(C)(C)[C@H]12</smiles>

50

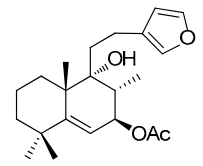

47

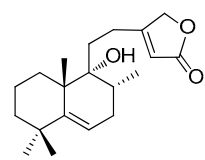

51<smiles>CC(=O)OC1CCC2(C)[C@@H](CC[C@H](C)[C@H]2C)[C@H]1Cc1ccoc1</smiles>

48<smiles>CC1=C(CCC(C)C(=O)CCC2=CC(=O)OC2)C(C)(C)CCC1</smiles>

52

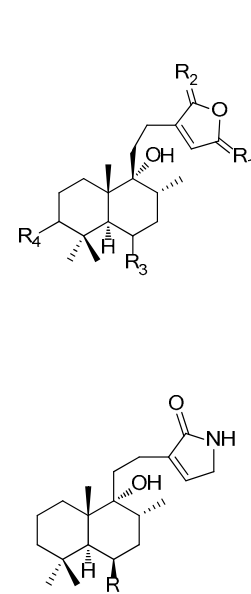

$73 \mathrm{R}=\mathrm{OAC}$

$74 \mathrm{R}=\mathrm{H}$

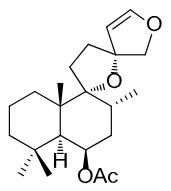

78

$53 \quad R_{1}=O \quad R_{2}=H_{2} \quad R_{3}=\beta-O A c \quad R_{4}=H$ $54 \quad R_{1}=H_{2} \quad R_{2}=O \quad R_{3}=\beta-O A C \quad R_{4}=H$

$55 \mathrm{R}_{1}=\mathrm{H}_{2} \quad \mathrm{R}_{2}=\mathrm{O} \quad \mathrm{R}_{3}=\mathrm{a}-\mathrm{OAC} \quad \mathrm{R}_{4}=\mathrm{H}$ $56 \mathrm{R}_{1}=\mathrm{H}, \mathrm{OCH}_{3} \quad \mathrm{R}_{2}=\mathrm{O} \mathrm{R}_{3}=\beta-\mathrm{OAC} \mathrm{R}_{4}=\mathrm{H}$ $57 R_{1}=O \quad R_{2}=H, O H R_{3}=\beta-O A c R_{4}=H$ $158 R_{1}=O R_{2}=H, O C H_{3} \quad R_{3}=\beta-O A c R_{4}=H$ $59 \mathrm{R}_{1}=\mathrm{O} \quad \mathrm{R}_{2}=\mathrm{H}_{2} \quad \mathrm{R}_{3}=\mathrm{R}_{4}=\mathrm{H}$ $60 \quad R_{1}=O \quad R_{2}=H_{2} \quad R_{3}=\beta-O H \quad R_{4}=H$ $61 \mathrm{R}_{1}=\mathrm{H}_{2} \quad \mathrm{R}_{2}=\mathrm{O} \quad \mathrm{R}_{3}=\mathrm{R}_{4}=\mathrm{H}$ $62 \mathrm{R}_{1}=\mathrm{H}_{2} \mathrm{R}_{2}=\mathrm{O} \mathrm{R}_{3}=\mathrm{H} \mathrm{R}_{4}=\beta-\mathrm{OH}$ $63 \mathrm{R}_{1}=\mathrm{H}_{2} \mathrm{R}_{2}=\mathrm{O} \mathrm{R}_{3}=\mathrm{H} \mathrm{R}_{4}=\beta-\mathrm{OAC}$ $64 \mathrm{R}_{1}=\mathrm{O} \mathrm{R}_{2}=\mathrm{H}_{2} \quad \mathrm{R}_{3}=\mathrm{H} \mathrm{R}_{4}=\beta-\mathrm{OAC}$

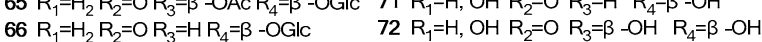

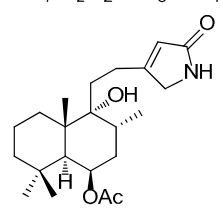

75

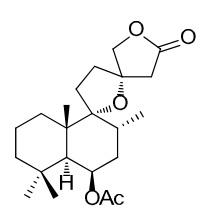

79<smiles>[R]c1cc(C([R])C[C@H]2C(=C)CC([R])[C@]3(C)[C@H]2[C@@H](C)CCC[C@H]3C)c([R7])o1</smiles>

$67 \mathrm{R}_{1}=\mathrm{H}_{2} \quad \mathrm{R}_{2}=\mathrm{O} \quad \mathrm{R}_{3}=\alpha-\mathrm{OH} \quad \mathrm{R}_{4}=\mathrm{H}$ $68 \mathrm{R}_{1}=\mathrm{O} \mathrm{R}_{2}=\mathrm{H}_{2} \mathrm{R}_{3}=\mathrm{B}-\mathrm{OH} \mathrm{R}_{4}=\mathrm{H}$

$69 \mathrm{R}_{1}=\mathrm{H}, \mathrm{OH} \mathrm{R}_{2}=\mathrm{O} \mathrm{R}_{3}=\alpha-\mathrm{OH} \mathrm{R}_{4}=\mathrm{H}$

$69 R_{1}=H, O H R_{2}=O R_{3}=\alpha-O H R_{4}=H$
$70 R_{1}=H, O H R_{2}=O \quad R_{3}=\beta-O H \quad R_{4}=H$

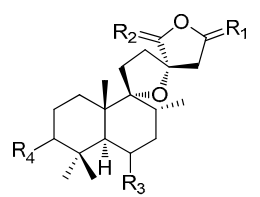

$82 \mathrm{R}_{1}=\mathrm{H}, \mathrm{OCH}_{3} \quad \mathrm{R}_{2}=\mathrm{O} \quad \mathrm{R}_{3}=\beta-\mathrm{OAc} \mathrm{R}_{4}=\mathrm{H}$

$83 \mathrm{R}_{1}=\mathrm{O} \quad \mathrm{R}_{2}=\alpha-\mathrm{OCH}_{3}, \beta-\mathrm{H} \mathrm{R}_{3}=\beta-\mathrm{OAc} \mathrm{R}_{4}=\mathrm{H}$

$84 \quad R_{1}=O \quad R_{2}=\alpha-H, \beta-O_{3} \quad R_{3}=\beta-O A c R_{4}=H$

$85 \mathrm{R}_{1}=\mathrm{O} \quad \mathrm{R}_{2}=\mathrm{H}_{2} \mathrm{R}_{3}=\beta-\mathrm{OH} \mathrm{R}_{4}=\mathrm{H}$

$86 \mathrm{R}_{1}=\mathrm{H}_{2} \mathrm{R}_{2}=\mathrm{H}, \mathrm{OH} \mathrm{R}_{3}=\beta-\mathrm{OAc} \mathrm{R}_{4}=\mathrm{H}$

$87 \mathrm{R}_{1}=\alpha-\mathrm{OCH}_{3}, \beta-\mathrm{H}_{2}=\mathrm{H}_{2} \mathrm{R}_{3}=\beta-\mathrm{OAc} \mathrm{R}_{4}=\mathrm{H}$

$88 R_{1}=\alpha-H, \beta-O C H_{3} \quad R_{2}=H_{2} \quad R_{3}=\beta-O A c R_{4}=H$

$89 \mathrm{R}_{1}=\mathrm{R}_{2}=\alpha-\mathrm{OCH}_{3}, \beta-H \mathrm{R}_{3}=\beta-\mathrm{OAc} \mathrm{R}_{4}=\mathrm{H}$

$90 \mathrm{R}_{1}=\mathrm{R}_{2}=\alpha-\mathrm{H}, \beta-\mathrm{OCH}_{3} \mathrm{R}_{3}=\beta-\mathrm{OAc} \mathrm{R}_{4}=\mathrm{H}$

$91 \mathrm{R}_{1}=\alpha-\mathrm{H}, \beta-\mathrm{OCH}_{3} \mathrm{R}_{2}=\alpha-\mathrm{OCH}_{3}, \beta-\mathrm{H} \mathrm{R}_{3}=\beta-\mathrm{OAC} \mathrm{R}_{4}=\mathrm{H}$

$92 \mathrm{R}_{1}=\alpha-\mathrm{OCH}_{3}, \beta-\mathrm{H} \mathrm{R} \mathrm{R}_{2}=\alpha-\mathrm{H}, \beta-\mathrm{OCH}_{3} \mathrm{R}_{3}=\beta-\mathrm{OAC} \mathrm{R}_{4}=\mathrm{H}$

$93 \mathrm{R}_{1}=\mathrm{R}_{2}=\alpha-\mathrm{OCH}_{3}, \beta-\mathrm{H} \mathrm{R}_{3}=\mathrm{R}_{4}=\mathrm{H}$

$94 \mathrm{R}_{1}=\mathrm{R}_{2}=\alpha-\mathrm{H}, \beta-\mathrm{OCH}_{3} \mathrm{R}_{3}=\mathrm{R}_{4}=\mathrm{H}$

$95 \mathrm{R}_{1}=\alpha-\mathrm{H}, \beta-\mathrm{OCH}_{3} \mathrm{R}_{2}=\alpha-\mathrm{OCH}_{3}, \beta-\mathrm{H} \mathrm{R}_{3}=\mathrm{R}_{4}=\mathrm{H}$

$96 \mathrm{R}_{1}=\mathrm{O} \mathrm{R}_{2}=\mathrm{H}, \mathrm{OH} \mathrm{R}_{3}=\mathrm{H} \mathrm{R}_{4}=\beta-\mathrm{OAC}$

$97 \mathrm{R}_{1}=\mathrm{O} \mathrm{R}_{2}=\mathrm{H}, \beta-\mathrm{OCH}_{3} \quad \mathrm{R}_{3}=\mathrm{H} \mathrm{R}_{4}=\beta-\mathrm{OAC}$

$98 \mathrm{R}_{1}=\mathrm{H}_{2} \mathrm{R}_{2}=\mathrm{H}, \alpha-\mathrm{OH} \mathrm{R}_{3}=\mathrm{H} \mathrm{R}_{4}=\beta-\mathrm{OAc}$

$99 \mathrm{R}_{1}=\mathrm{R}_{2}=\alpha-\mathrm{OCH}_{3}, \beta-\mathrm{H} \mathrm{R_{3 }}=\mathrm{H} \mathrm{R_{4 }}=\beta-\mathrm{OAC}$

$100 \mathrm{R}_{1}=\alpha-\mathrm{H}, \beta-\mathrm{OCH}_{3} \mathrm{R}_{2}=\alpha-\mathrm{OCH}_{3}, \beta-H \mathrm{R}_{3}=H \mathrm{R}_{4}=\beta-\mathrm{OAC}$<smiles>CC1=CC[C@H]2[C@@H](C)CCC[C@H]2C[C@H]1C[C@H](O)C1=CC(=O)O[C@@H]1O</smiles>

76

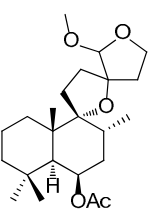

80

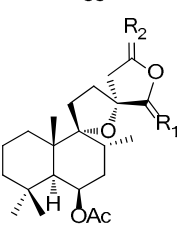

$101 \mathrm{R}_{1}=\mathrm{O} \quad \mathrm{R}_{2}=\mathrm{H}, \mathrm{OCH}_{3}$ $102 \mathrm{R}_{1}=\alpha-\mathrm{OCH}_{3}, \beta-\mathrm{H}_{2} \mathrm{R}_{2}=\mathrm{O}$ $103 \mathrm{R}_{1}=\alpha-\mathrm{H}, \beta-\mathrm{OCH}_{3} \quad \mathrm{R}_{2}=\mathrm{O}$ $104 \mathrm{R}_{1}=\mathrm{H}_{2} \mathrm{R}_{2}=\alpha-\mathrm{OCH}_{3}, \beta-\mathrm{H}$ $105 \mathrm{R}_{1}=\mathrm{H}_{2} \mathrm{R}_{2}=\alpha-\mathrm{H}, \beta-\mathrm{OCH}_{3}$<smiles>C[C@@H]1CC=C2C(C)(C)CCC[C@]2(C)[C@@]12CC[C@@]1(COC(=O)C1)O2</smiles>

77

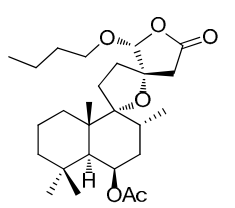

81

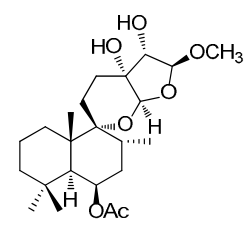

106

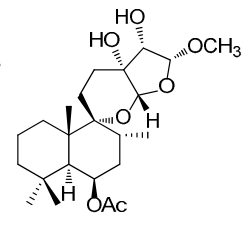

107

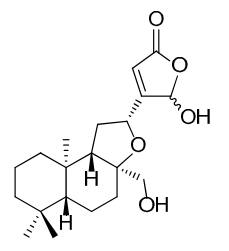

109

Figure 3. Cont. 
<smiles>C=C1CC[C@H](C)C2(C)CCC[C@@H](C)C12CC(O)/C=C(\C)C(=O)O</smiles>

110

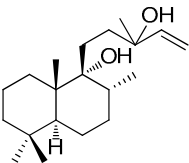

114

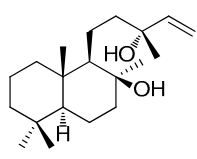

118

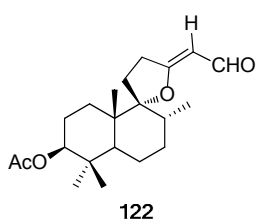

122

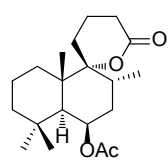

132<smiles>C=C(O)CC(=O)CC1C(=O)CC[C@@H]2CCCCC12C</smiles>

111

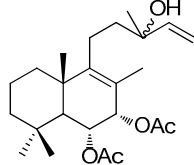

112<smiles>C=CC(C)(O)CC[C@@H]1[C@@](C)(O)CC[C@@H]2C(C)(C)CCC[C@@]12C</smiles>

116

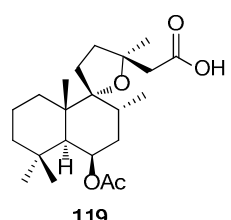

119
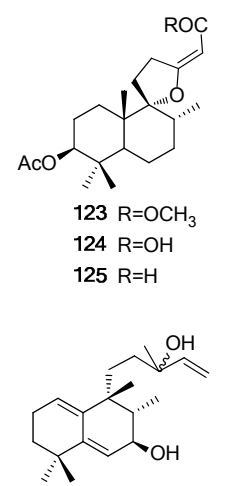

133<smiles>CC1CCC2CCCCC23CCCOC3CC1</smiles>

120

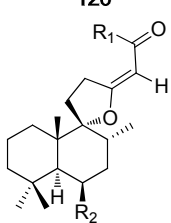

$126 \mathrm{R}_{1}=\mathrm{R}_{2}=\mathrm{H}$ $127 \mathrm{R}_{1}=\mathrm{H} \mathrm{R}_{2}=\mathrm{OAC}$

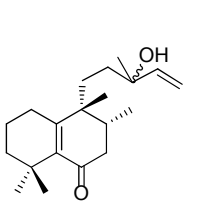

134

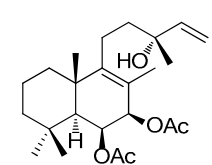

113<smiles>C=CC1(C)CCC2C(C)(O)CC[C@H]3[C@@H](C)CCC[C@@]23C1(C)C</smiles>

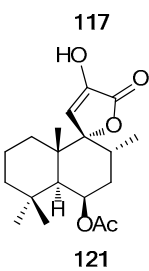

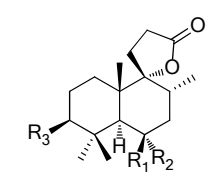

$128 \mathrm{R}_{1}=\mathrm{H} \mathrm{R}_{2}=\mathrm{OAc} \mathrm{R}_{3}=\mathrm{H}$ $129 \mathrm{R}_{1}=\mathrm{OAc} \mathrm{R}_{2}=\mathrm{R}_{3}=\mathrm{H}$ $130 \mathrm{R}_{1}=\mathrm{R}_{2}=\mathrm{R}_{3}=\mathrm{H}$ $131 \mathrm{R}_{1}=\mathrm{R}_{2}=\mathrm{H} \mathrm{R}_{3}=\mathrm{OAC}$<smiles>C=CC(C)(O)C[C@H]1[C@@H]2CCC(OC(C)=O)C(C)(C)C2=CC[C@H]1C</smiles>

135<smiles></smiles><smiles>[R]C1C2=C(CCCC2(C)C)[C@](C)(CC)[C@@H](C)C1[R]</smiles>

$137 \mathrm{R}_{1}=\mathrm{R}_{2}=\mathrm{OH}$ $138 \mathrm{R}_{1}=\mathrm{R}_{2}=\mathrm{OAC}$

$139 \mathrm{R}_{1}=\mathrm{OAc} \mathrm{R}_{2}=\mathrm{H}$

$140 \mathrm{R}_{1}=\mathrm{H} \mathrm{R}_{2}=\mathrm{OAc}$<smiles>[R]c1cc2c(cc1C(C)C)CC[C@H]1[C@@H](C)[C@H]([R])CC[C@@]21C</smiles>

$142 \mathrm{R}_{1}=\mathrm{H} \mathrm{R}_{2}=\mathrm{OH}$ $143 \mathrm{R}_{1}=\mathrm{OH} \mathrm{R} \mathrm{R}_{2}=\mathrm{H}$<smiles>CC(C)c1ccc2c(c1)C[C@@H](O)[C@]1(C)[C@@H](C)CCC[C@@]21C</smiles>
$140 \mathrm{R}_{1}=\mathrm{H}_{1}=\mathrm{OCH}_{2}=\mathrm{OAC}$
$\mathrm{R}_{2}=\mathrm{H}$<smiles>CC(C)c1ccc2c(c1)C(=O)CC1C2(C)CC[C@@H](O)C1(C)C</smiles><smiles>CC(C)C1=CC=C2[C@@H](CC[C@H]3[C@H](C)CCC[C@@]23C)C1</smiles>

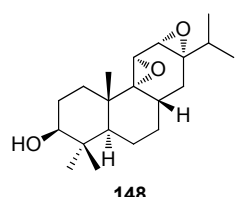

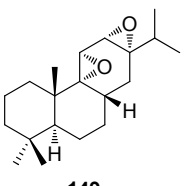<smiles>CC(C)c1ccc2c(c1)CCC1CCCC21CO</smiles>

147

148

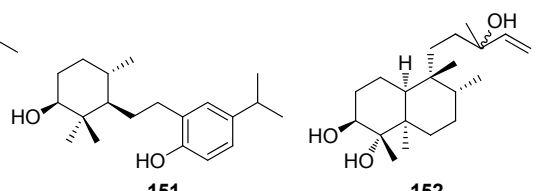<smiles>C=CC1(C)CC[C@H]2C(C)=CC[C@]3(C)C(C)CCC[C@H]3[C@H]2C1</smiles>

153

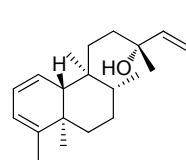

154

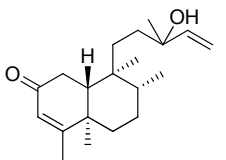

155

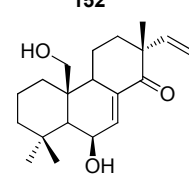

156

Figure 3. Structures of diterpenoids 43-156 isolated from Vitex L. 


\subsection{Triterpenoids}

The triterpenoids isolated from the genus are mainly pentacyclic triterpenoids, consisting of oleanane-type (compounds 157-183), ursane-type (compounds 184-201), norursane-type (compounds 202-203), lupane-type (compounds 204-208) and friedelane-type (209). Only a few (compounds 162, 178, 181, 182, 195, 196) are triterpenoid glycosides $[67,68]$. Among them, cannabifolins A (184) and B (183) are the first examples of 12,19-epoxyursane- and oleanane-type triterpenoids and are rare natural pentacyclic triterpenoids with cis-fused C/D rings [69]. Tetracyclic triterpenoids like the 9-epi-cucurbitane-type $\mathbf{2 1 0}$ also has been isolated [43]. The names of these triterpenoids and their structures are listed in Table 4 and shown in Figure 4, respectively.

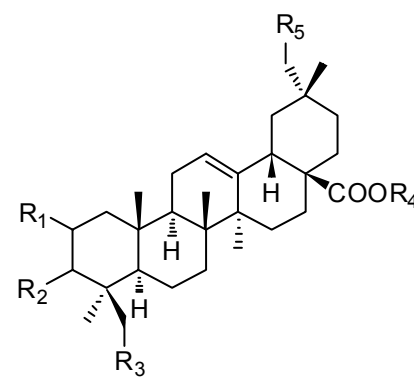

$157 \mathrm{R}_{1}=\mathrm{H} \mathrm{R} \mathrm{R}_{2}=\mathrm{B}-\mathrm{OH} \mathrm{R}_{3}=\mathrm{R}_{4}=\mathrm{R}_{5}=\mathrm{H}$

$158 \mathrm{R}_{1}=\mathrm{a}-\mathrm{OH} \mathrm{R}_{2}=\mathrm{B}-\mathrm{OH} \mathrm{R}_{3}=\mathrm{R}_{4}=\mathrm{R}_{5}=\mathrm{H}$

$159 \mathrm{R}_{1}=\mathrm{R}_{2}=\mathrm{a}-\mathrm{OH} \mathrm{R}_{3}=\mathrm{R}_{4}=\mathrm{R}_{5}=\mathrm{H}$

$160 \mathrm{R}_{1}=\mathrm{R}_{2}=\mathrm{a}-\mathrm{OH} \quad \mathrm{R}_{3}=\mathrm{OH} \mathrm{R}_{4}=\mathrm{R}_{5}=\mathrm{H}$

$161 \mathrm{R}_{1}=\mathrm{a}-\mathrm{OH} \mathrm{R}_{2}=\mathrm{B}-\mathrm{OH} \mathrm{R}_{3}=\mathrm{OH} \mathrm{R}_{4}=\mathrm{R}_{5}=\mathrm{H}$

$162 R_{1}=R_{2}=a-O H \quad R_{3}=O H \quad R_{4}=\beta-D-G l c R_{5}=H$

$163 R_{1}=R_{2}=a-O H \quad R_{3}=R_{4}=H R_{5}=O H$

$164 \mathrm{R}_{1}=\mathrm{H} \mathrm{R} \mathrm{R}_{2}=$ B-OAC $\mathrm{R}_{3}=\mathrm{R}_{4}=\mathrm{R}_{5}=\mathrm{H}$

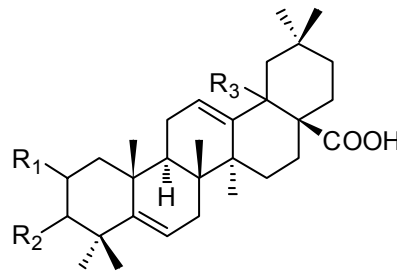

$170 \mathrm{R}_{1}=\mathrm{H} \mathrm{R} \mathrm{R}_{2}=ß-\mathrm{OH} \quad \mathrm{R}_{3}=\mathrm{H}$

$171 \mathrm{R}_{1}=\mathrm{R}_{2}=\mathrm{a}-\mathrm{OH} \mathrm{R}_{3}=\mathrm{H}$

$172 R_{1}=\beta-O A c R_{2}=a-O A c R_{3}=H$

$173 R_{1}=a-O A c \quad R_{2}=ß-O A c R_{3}=O H$

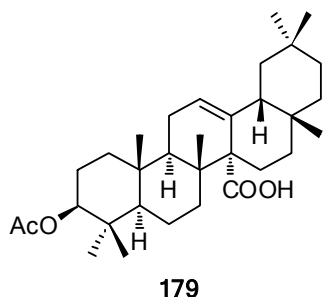

179

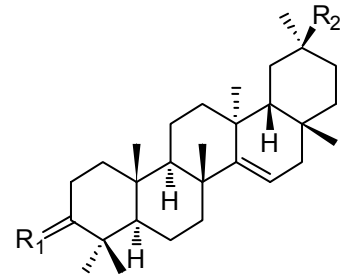

$174 \mathrm{R}_{1}=\mathrm{a}-\mathrm{H}, \mathrm{B}-\mathrm{OH} \mathrm{R}_{2}=\mathrm{CH}_{3}$

$175 \mathrm{R}_{1}=\mathrm{O} \mathrm{R}_{2}=\mathrm{CH}_{3}$

$176 \mathrm{R}_{1}=\mathrm{O} \mathrm{R}_{2}=\mathrm{CHO}$

$165 \mathrm{R}_{1}=\mathrm{H} \mathrm{R}_{2}=ß-\mathrm{OH} \mathrm{R}_{3}=\mathrm{R}_{4}=\mathrm{H}$

$166 \mathrm{R}_{1}=\mathrm{R}_{2}=\mathrm{a}-\mathrm{OH} \mathrm{R}_{3}=\mathrm{H} \mathrm{R}_{4}=\mathrm{CH}_{3}$

$167 \mathrm{R}_{1}=\mathrm{R}_{2}=\mathrm{a}-\mathrm{OH} \mathrm{R}_{3}=\mathrm{R}_{4}=\mathrm{H}$

$168 \mathrm{R}_{1}=\mathrm{a}-\mathrm{OH} \mathrm{R}_{2}=\mathrm{B}-\mathrm{OH} \mathrm{R}_{3}=\mathrm{a}-\mathrm{OH} \mathrm{R}_{4}=\mathrm{H}$

$169 \mathrm{R}_{1}=\mathrm{a}-\mathrm{OH} \mathrm{R}_{2}=ß-\mathrm{OH} \mathrm{R}_{3}=\mathrm{R}_{4}=\mathrm{H}$

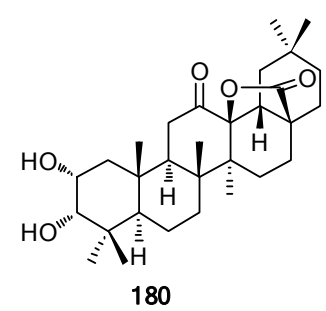

Figure 4. Cont. 


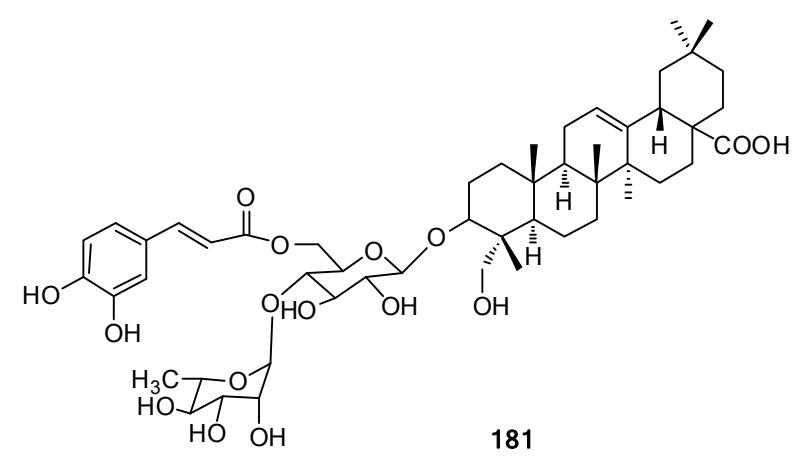

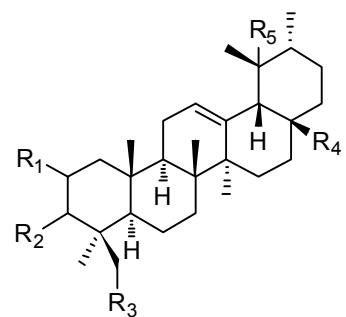

$185 R_{1}=\mathrm{H} \mathrm{R}_{2}=ß-\mathrm{OH} \mathrm{R}_{3}=\mathrm{H} \mathrm{R}_{4}=\mathrm{COOH} \mathrm{R}_{5}=\mathrm{H}$ $186 \mathrm{R}_{1}=\mathrm{H} \mathrm{R}_{2}=\mathrm{a}-\mathrm{OH} \mathrm{R}_{3}=\mathrm{H} \mathrm{R}_{4}=\mathrm{COOH} \mathrm{R}_{5}=\mathrm{H}$ $187 \mathrm{R}_{1}=\mathrm{a}-\mathrm{OH} \mathrm{R}_{2}=\mathrm{B}-\mathrm{OH} \mathrm{R}_{3}=\mathrm{H} \mathrm{R}_{4}=\mathrm{COOH} \mathrm{R}_{5}=\mathrm{H}$ $188 \mathrm{R}_{1}=\mathrm{R}_{2}=\mathrm{a}-\mathrm{OH} \mathrm{R}_{3}=\mathrm{H} \mathrm{R}_{4}=\mathrm{COOH} \mathrm{R}_{5}=\mathrm{H}$ $189 R_{1}=H R_{2}=B-O A c \quad R_{3}=H \quad R_{4}=C O O H \quad R_{5}=H$ $190 R_{1}=H_{2}=B-O H R_{3}=\mathrm{H} \mathrm{R}_{4}=\mathrm{CH}_{3} \mathrm{R}_{5}=\mathrm{H}$ $191 \mathrm{R}_{1}=\mathrm{H} \mathrm{R}_{2}=\mathrm{B}-\mathrm{OH} \mathrm{R}_{3}=\mathrm{H} \mathrm{R}_{4}=\mathrm{CH}_{2} \mathrm{OH} \mathrm{R}_{5}=\mathrm{H}$ $192 R_{1}=a-O H \quad R_{2}=ß-O H \quad R_{3}=\mathrm{H}_{4}=\mathrm{COOH} R_{5}=\mathrm{OH}$ $193 \mathrm{R}_{1}=\mathrm{R}_{2}=\mathrm{a}-\mathrm{OH} \mathrm{R}_{3}=\mathrm{OH} \mathrm{R}_{4}=\mathrm{COOH} \mathrm{R}_{5}=\mathrm{H}$ $194 \mathrm{R}_{1}=\mathrm{R}_{2}=\mathrm{a}-\mathrm{OH} \mathrm{R}_{3}=\mathrm{H} \mathrm{R}_{4}=\mathrm{COOH} \mathrm{R}_{5}=\mathrm{OH}$

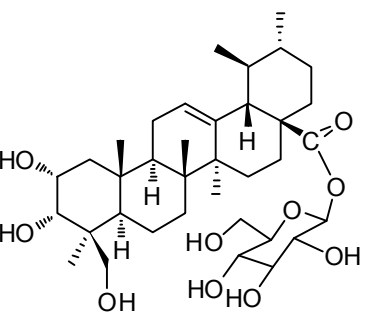

195

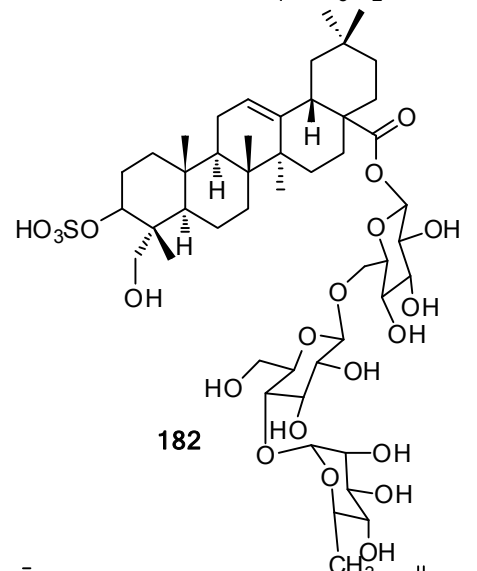

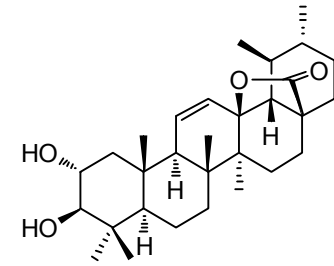

201

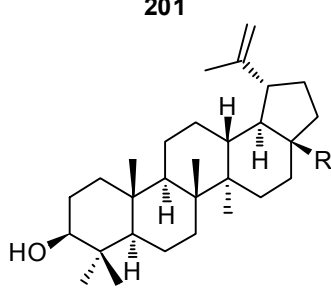

$204 \mathrm{R}=\mathrm{CH}_{3}$ $205 \mathrm{R}=\mathrm{COOH}$

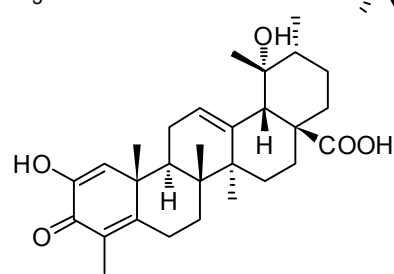

202

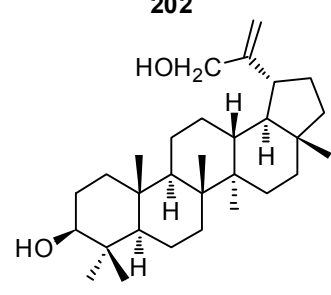

206

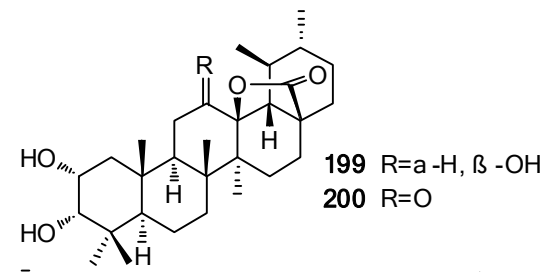

$196 \mathrm{R}_{1}=\mathrm{OH} \quad \mathrm{R}_{2}=\mathrm{OGlc}$

$197 \mathrm{R}_{1}=\mathrm{R}_{2}=\mathrm{OH}$

$198 \mathrm{R}_{1}=\mathrm{H} \mathrm{R}_{2}=\mathrm{OH}$

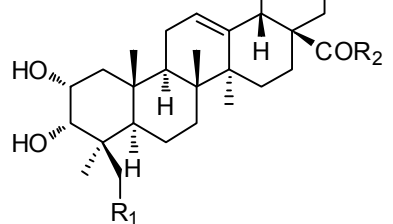<smiles>Cc1ccc2c(c1C)C1=CC[C@H]3C4(C)C[C@H](O)[C@@H](O)C(C)(C)[C@H]4CC[C@]3(C)[C@H]1CC2</smiles>

203

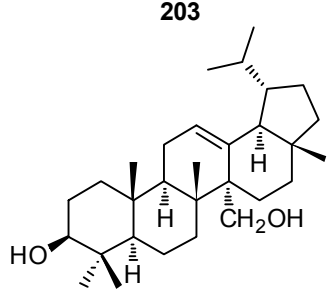

207<smiles>CC(=O)C1CCC2(C(=O)O)CC[C@H]3C4CC[C@H]5[C@H](C)[C@@H](O)CC[C@]5(C)C4CC[C@]3(C)[C@H]12</smiles>

208

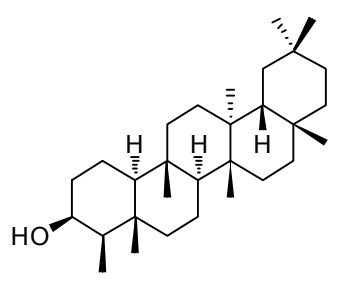

209

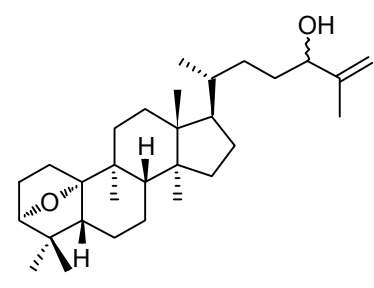

210

Figure 4. Structures of triterpenoids 157-210 isolated from Vitex L. 
Table 4. Triterpenoids $\mathbf{1 5 7 - 2 1 0}$ isolated from plants of Vitex L.

\begin{tabular}{|c|c|c|c|c|}
\hline Type & No. & Compound Name & Source & Reference \\
\hline \multirow{27}{*}{ Oleanane } & 157 & Oleanolic acid & $\mathrm{a}, \mathrm{b}$ & {$[54,68,70,71]$} \\
\hline & 158 & Maslinic acid & $\mathrm{a}, \mathrm{c}, \mathrm{f}$ & {$[33,54,72,73]$} \\
\hline & 159 & 3-Epimaslinic acid & $a, c, f, h$ & {$[33,51,69,71,73]$} \\
\hline & 160 & $2 \alpha, 3 \alpha, 24$-Trihydroxyolean-12-en-28-oic acid & $a, b, h$ & {$[69,72,74]$} \\
\hline & 161 & $2 \alpha, 3 \beta, 24$-Trihydroxyolean-12-en-28-oic acid & $\mathrm{b}$ & [74] \\
\hline & 162 & Vulgarsaponin A & a & [67] \\
\hline & 163 & Cannabifolin F & $\mathrm{h}$ & [69] \\
\hline & 164 & Oleanolic acid acetate & a & [75] \\
\hline & 165 & Hederagenin & $\mathrm{b}$ & [68] \\
\hline & 166 & $2 \alpha, 3 \alpha, 23$-Trihydroxyolean-12-en-28-oic acid methyl ester & a & [71] \\
\hline & 167 & $2 \alpha, 3 \alpha, 23$-Trihydroxyolean-12-en-28-oic acid & a & [71] \\
\hline & 168 & $2 \alpha, 3 \beta, 19 \alpha, 23$-Tetrahydroxyolean-12-en-28-oic acid & $\mathrm{a}$ & [72] \\
\hline & 169 & $2 \alpha, 3 \beta, 23$-Trihydroxyolean-12-en-28-oic acid & $\mathrm{a}$ & [72] \\
\hline & 170 & $3 \beta$-Hydroxyolean-5,12-dien-28-oic acid & a & [76] \\
\hline & 171 & $2 \alpha, 3 \alpha$-Dihydroxyoleana-5,12-dien-28-oic acid & $\mathrm{a}$ & [77] \\
\hline & 172 & $2 \beta, 3 \alpha$-Diacetoxyoleana-5,12-dien-28-oic acid & a & [77] \\
\hline & 173 & $2 \alpha, 3 \beta$-Diacetoxy-18-hydroxyoleana-5,12-dien-28-oic acid & a & [77] \\
\hline & 174 & Taraxerol & $\mathrm{b}$ & [78] \\
\hline & 175 & Taraxerone & 1 & [79] \\
\hline & 176 & 3-Oxotaraxer-14-en-30-al & 1 & [79] \\
\hline & 177 & $\beta$-Amyrin & $\mathrm{a}, \mathrm{b}$ & {$[68,71]$} \\
\hline & 178 & $\beta$-Amyrin-3-O- $\beta$-D-glucopyranoside & $\mathrm{b}$ & [68] \\
\hline & 179 & $3 \beta$-Acetoxyolean-12-en-27-oic acid & a & {$[76,77]$} \\
\hline & 180 & Cannabifolin E & $\mathrm{h}$ & [69] \\
\hline & 181 & $\begin{array}{l}\text { 23-Hydroxy-3 } 3 \alpha-\left[O-\alpha \text {-L-rhamnopyranosyl-( }\left(1^{\prime \prime \prime} \rightarrow 4^{\prime \prime}\right)-O-\left[\beta-\mathrm{D}-\left(E-6^{\prime \prime}-\right.\right.\right. \\
\text { O-caffeoyl)-glucopyranosyl]-oxy]-olean-12-en-28-oic acid }\end{array}$ & $\mathrm{b}$ & {$[68]$} \\
\hline & 182 & $\begin{array}{l}\text { 23-hydroxy-3 } \alpha \text {-(O-sulfonyloxy)-olean-12-en-28-oic acid-28-O-[ } \alpha \text {-L- } \\
\text { rhamnopyranosyl-( }\left(1^{\prime \prime \prime} \rightarrow 4^{\prime \prime}\right)-O-\beta \text {-D-glucopyranosyl- }\left(1^{\prime \prime} \rightarrow 6^{\prime}\right)-O-\beta \text {-D-glucopyranosyl] ester }\end{array}$ & $\mathrm{b}$ & [68] \\
\hline & 183 & Cannabifolin B & $\mathrm{h}$ & [69] \\
\hline
\end{tabular}


Table 4. Cont.

\begin{tabular}{|c|c|c|c|c|}
\hline Type & No. & Compound Name & Source & Reference \\
\hline \multirow{18}{*}{ Ursane } & 184 & Cannabifolin A & $\mathrm{h}$ & [69] \\
\hline & 185 & Ursolic acid & $a-c, e, h$ & {$[49,53,54,69,72,73,78,80,81]$} \\
\hline & 186 & 3-Epiursolic acid & $\mathrm{b}, 1$ & {$[74,79]$} \\
\hline & 187 & Corosolic acid & $a-c, e, f, h, l$ & {$[33,53,67,69,73,79,80]$} \\
\hline & 188 & 3-Epicorosolic acid & $a-c, f, h$ & {$[28,33,51,69,73,78]$} \\
\hline & 189 & $3 \beta$-Acetoxyurs-12-en-28-oic acid & $\mathrm{b}$ & {$[49,82]$} \\
\hline & 190 & $\alpha$-Amyrin & $\mathrm{b}$ & [53] \\
\hline & 191 & Uvaol & $\mathrm{b}$ & [74] \\
\hline & 192 & Tormentic acid & $a, b, e, h$ & {$[69,72,78,83]$} \\
\hline & 193 & $2 \alpha, 3 \alpha, 24$-Trihydroxyurs-12-en-28-oic acid & $\mathrm{b}, \mathrm{c}$ & {$[73,74]$} \\
\hline & 194 & Euscaphic acid & $\mathrm{c}, \mathrm{h}$ & {$[69,73]$} \\
\hline & 195 & $2 \alpha, 3 \alpha, 24$-Trihydroxyurs-12-en-28-oic acid-28-O- $\beta$-D-glucopyranosyl ester & a & [67] \\
\hline & 196 & $2 \alpha, 3 \alpha, 24$-Trihydroxyurs-12,20(30)-dien-28-oic acid-28-O- $\beta$-D-glucopyranosyl ester & a & [67] \\
\hline & 197 & $2 \alpha, 3 \alpha, 24$-Trihydroxyurs-12,20(30)-dien-28-oic acid & $\mathrm{c}$ & [73] \\
\hline & 198 & $2 \alpha, 3 \alpha-$-Dihydroxyurs-12,20(30)-dien-28-oic acid & $\mathrm{h}$ & [69] \\
\hline & 199 & Cannabifolin C & $\mathrm{h}$ & [69] \\
\hline & 200 & Cannabifolin D & $\mathrm{h}$ & [69] \\
\hline & 201 & Ilelatifol D & $\mathrm{f}$ & [51] \\
\hline \multirow{2}{*}{ Norursane } & 202 & Negundonorin A & $\mathrm{a}$ & [28] \\
\hline & 203 & Negundonorin B & a & [28] \\
\hline \multirow{5}{*}{ Lupane } & 204 & Lupeol & 1 & [79] \\
\hline & 205 & Betulinic acid & $a, b, 1$ & {$[49,54,78,79,81]$} \\
\hline & 206 & Lup-20(29)-en-3 $\beta, 30$-diol & a & [54] \\
\hline & 207 & Obtusalin & a & [54] \\
\hline & 208 & Platanic acid & $\mathrm{b}$ & [82] \\
\hline Friedelane & 209 & Epifriedelinol & e & [84] \\
\hline 9-epi-Cucurbitane & 210 & $(24 R / S)-24$-Hydroxy-3 $\alpha, 10 \alpha$-epoxy-9-epi-cucurbita-25-ene & a & [43] \\
\hline
\end{tabular}




\section{Pharmacological Effects}

Terpenes isolated from Vitex plants have been evaluated for their anti-inflammatory, anti-tumor, antibacterial, antioxidant and other pharmacological effects, which provide potential explanations for their use in the treatment of various diseases in folk medicine. It was proved that terpenes were the principal active constituents for the aforementioned effects. A detailed summary of their pharmacological studies is given below.

\subsection{Anti-Inflammatory Activity}

Many plants from Vitex genus have been used for the treatment of inflammatory diseases. And pharmacological studies have also shown that some terpenes isolated from the genus have significant anti-inflammatory effects. Agnuside (3) exerted significant anti-inflammatory activity using carrageenan-, histamine- and dextran-induced acute inflammation models in rats. The inhibitory effect seemed independent of activation of the pituitary-adrenal axis because the inhibition effects against carrageenan-induced oedema in normal and adrenalectomized rats after oral administration of agnuside (3) were highly comparable. Furthermore, oral administration of agnuside (3) to arthritic rats can decrease the levels of intracellular interleukin-17 (IL-17) in lymphocytes with values of $12.17 \%$ and $11.04 \%$ at doses of 6.12 and $12.5 \mathrm{mg} / \mathrm{kg}$, compared with non-agnuside-fed control groups $19.71 \%$ [20]. Twenty-four different compounds were isolated from V. rotundifolia by Lee et al., and their anti-inflammatory activities were tested by the Griess method. The results revealed that five diterpenoids (compounds 57, 61, 106, 141, 138) significantly inhibited nitric oxide (NO) production in lipopolysaccharide (LPS)-stimulated RAW 264.7 cells with the $\mathrm{IC}_{50}$ values of 11.3, 16.4, 17.2, 22.2 and $24.5 \mu \mathrm{M}$ respectively, while the positive control aminoguanidine being $16.6 \mu \mathrm{M}$ [45]. Li et al. [69] isolated fourteen triterpenoids from V. negundo var. cannabifolia, of which five compounds $(\mathbf{1 9 2}, \mathbf{1 9 8}$, 159, 199, and 160) demonstrated moderate inhibitory effects on NO production, with $\mathrm{IC}_{50}$ values of $24.9 \pm 4.6,26.1 \pm 3.6,27.7 \pm 3.3,34.0 \pm 4.1,40.5 \pm 4.9 \mu \mathrm{M}$, respectively. Zheng et al. [37] have isolated nine diterpenoids (compounds 97, 98, 123-125, 131, 145, 151, 156) from the seeds of $V$. negundo. Among these, negundoin C (125) and negundoin E (98) showed the most significant inhibitory effects on NO production using LPS-stimulated RAW 264.7 cells, with $\mathrm{IC}_{50}$ values of 0.12 and $0.23 \mu \mathrm{M}$, respectively, compared with the positive control indomethacin at $45.51 \mu \mathrm{M}$. Additionally, the authors demonstrated the protein expressions of cyclooxygenase-2 (COX-2) and inducible nitric oxide synthetase (iNOS) with western blot analysis to describe the possible mechanism of their anti-inflammatory activity, and it was an interesting finding that the level of COX-2 protein and iNOS protein were decreased by $\mathbf{9 8}$ and $\mathbf{1 2 5}$.

\subsection{Anti-Tumor Activity}

It is also worth mentioning that some terpenes of the Vitex genus possess significant anti-tumor activities against several cancer cell lines. Wu et al. [6] isolated ten diterpenoids $\mathbf{3 4}, \mathbf{4 8}, \mathbf{5 3}, \mathbf{5 4}, \mathbf{7 9}$, 80, 137-140, including three new compounds 34, 48, 137, from $V$. trifolia L. All compounds were tested for their inhibitory effects on HeLa cell proliferation with the MTT assay, and their $\mathrm{IC}_{50}$ values ranged from $4.9 \pm 0.5$ to $28.7 \pm 1.3 \mu \mathrm{M}$. Furthermore, vitetrifolin I (137) exhibited significant inhibition effect with an $\mathrm{IC}_{50}$ value of $4.9 \pm 0.5 \mu \mathrm{M}$, and induced cell cycle $\mathrm{G}_{0} / \mathrm{G}_{1}$ phase arrest and apoptosis of HeLa cells. Six terpenes 35, 37, 122, 188, 202, 203 were isolated from $V$. negundo and evaluated for their cytotoxicities against four cancer cell lines using the MTT method. The results revealed that negunfurol (35) was the most active compound against HL-60, with an $\mathrm{IC}_{50}$ value of $0.94 \pm 0.26 \mu \mathrm{g} / \mathrm{mL}$ and negundonorin A (202) was highly cytotoxic to ZR-75-30 cells with an $\mathrm{IC}_{50}$ value of $0.56 \pm 0.19 \mu \mathrm{g} / \mathrm{mL}$ [28]. Mahesh et al. [35] isolated six diterpenoids 43, 53, 74, 75, 120, 138, including a new diterpenoid alkaloid 74, from V. agnus-castus. All compounds were evaluated for their cytotoxicities against the K562 cell line. The $\mathrm{IC}_{50}$ values ranged from 0.70 to $6.72 \mu \mathrm{g} / \mathrm{mL}$, and compound 74 was the most cytotoxic, with an $\mathrm{IC}_{50}$ value of $0.70 \mu \mathrm{g} / \mathrm{mL}$, compared with the 
positive control cisplatin at $1.10 \mu \mathrm{g} / \mathrm{mL}$. Corlay et al. [55] isolated nine labdane-type diterpenoids 67-72, 109-111 from $V$. vestita. All the diterpenoids except vitexolin A (110) were cytotoxic against the HCT-116 and MRC-5 cancer cell lines to some extent.

\subsection{Antibacterial and Antifungal Activities}

According to references $[44,55,59,84]$, some terpenes in the Vitex genus possess significant antibacterial and antifungal activities. Vitexilactone $C(49)$ showed weak antibacterial activity against Bacillus subtilis, Escherichia coli and Micrococcus tetragenus at the same minimum inhibitory concentration (MIC) value of $500 \mu \mathrm{g} / \mathrm{mL}$ [44]. The diterpenoid vitexolide A (69) isolated from $V$. vestita showed the most potent antibacterial activity against $46 \mathrm{Gram}$-positive strains compared with other diterpenoids 67, 68, 70, 109, 111. The MIC values ranged from 6 to $96 \mu \mathrm{M}$ [55]. Epifriedelinol (209) is a pentacyclic triterpenoid isolated from Vitex peduncularis by bioassay guided separation. Its antibacterial activity was tested against 12 strains of Gram positive and Gram negative bacteria. The MIC values were in the range of $6.25-50 \mu \mathrm{g} / \mathrm{mL}$. The minimum bactericidal concentration (MBC) values were in the range of 12.5-100 $\mu \mathrm{g} / \mathrm{mL}$ [84]. Additionally, negundol (96), a labdane-type diterpeoid isolated from the seeds of $V$. negundo exhibited antifungal activity against Candida albicans $\left(\mathrm{MIC}_{80}: 64 \mu \mathrm{g} / \mathrm{mL}\right.$ ), Cryptococcus neoformans $\left(\mathrm{MIC}_{80}: 16 \mu \mathrm{g} / \mathrm{mL}\right.$ ) and Trichophyton rubrum ( $\mathrm{MIC}_{80}: 32 \mu \mathrm{g} / \mathrm{mL}$ ) [59].

\subsection{Antioxidant Activity}

Results from different studies have demonstrated that many terpenes in the Vitex genus have significant antioxidant activites. Sridhar et al. [13] isolated six new acylated iridoid glucosides (compounds 17-22) from V. altissima, and each compound was tested for its superoxide radical-scavenging activity using the McCord and Fridovich method and 1-diphenyl-2-picrylhydrazyl $(\mathrm{DPPH})$ radical-scavenging effect with the Lamaison method. The results showed three compounds 18-20 exhibiting significant antioxidant activity by both methods. Tiwari et al. [21] isolated iridoid glucosides agnuside (3), negundoside (23) and 6'-O-p-hydroxybenzoyl mussaenosidic acid (12) from $V$. trifolia. Compounds 3, 23 and 12 showed DPPH radical scavenging avtivities with $\mathrm{IC}_{50}$ values of $9.81,9.96$ and $10.31 \mu \mathrm{g}$, respectively, and also effectively inhibited NO radical at $\mathrm{IC}_{50}$ values of 12.90, 16.25 and $13.51 \mu \mathrm{g}$. Ferruginol (142), an abietane-type diterpenoid isolated from V. rotundifolia showed higher antioxidant activity than 3-tert-butyl-4-hydroxyansiole (BHA) using the ferric thiocyanate method. Futhermore, it has stronger DPPH radical scavenging effect equivalent to half that of L-cysteine [58].

\subsection{Other Pharmacological Activities}

Additionally, some of the terpenes also have analgesic, endocrinological, anti-hyperglycemic, antifeedant effects, etc. Okuyama et al. [15] verified the analgesic effect of two iridoids agnuside (3) and 10-O-vanilloylaucubin (4) by the acetic acid induced writhing test in mice. At a dose of $50 \mathrm{mg} / \mathrm{kg}$ compounds 3 and 4 exerted analgesic effects of $56 \%(p<0.001)$ and $20 \%(p<0.05)$, respectively. Extracts of V. agnus-castus have been used for amelioration of premenopausal syndrome, especially mastodynia, which were most likely caused by hypersecretion of prolactin. The proposed mechanism of action was due to dopaminergic and estrogenic principle. The mixture of clerodane-type diterpenoids (BNO-diterpenoids), isolated from 70\% ethanolic extract of $V$. agnus-castus, showed the highest dopaminergic activity by reducing cyclic AMP (cAMP) formation and prolactin secretion [41]. Sundarama et al. [26] obtained the iridoid glucoside 23 from leaves of $V$. negundo, which could reduce the levels of blood glucose and glycoproteins, and increase the level of plasma insulin in streptozotocin diabetic rats. Compound 23 also showed anti-hyperlipidemic activity [27]. Additionally, hepatoprotective activity of some terpenes (compounds 23, 164) from genus Vitex plants was discovered by Indian scholars $[24,75,85]$. Ursolic acid (185) and betulinic acid (205) showed antifeedant activity against the larvae of Achoea janata [81]. 


\section{Conclusions}

In this review, we summarize the research progress on terpenes of the genus Vitex and their pharmacology. These findings indicate that this genus is a valuable source of bioactive molecules. Phytochemical and pharmacological studies of the compounds isolated from the genus Vitex have attracted more attention in recent years. Terpenes, including monoterpenoids, sesquiterpenoids, diterpenoids and triterpenoids were identified as the main chemical constituents of this genus. From the literature, there are approximately 250 species in the genus [1], but studies on terpenes had been done to some extent on only 12 species [9-21,30,55,56,62]. Considering the many bioactive terpenes isolated from the plants in this genus, further investigations on terpenes and their pharmacological effects of the other species are very necessary. In the pharmacology domain, most of the isolated terpenes have been evaluated for various activities in vitro without being further tested in vivo. Thus the promising pharmacological activities should be confirmed by in vivo assay using diverse rat models to prove them. In addition, taking into account their therapeutic efficiency, validating the relationships between chemical constituents, pharmacological effects and traditional uses of plants in this genus is still remains a fundamental task, and should be paid more attention to.

Acknowledgments: This work was supported by the National High Technology Research and Development Program of China (863 Program) (2013AA093001), the National Natural Science Foundation of China (81303142), the National Science Foundation for Post-doctoral Researchers (2015M570231) and the Tianjin City High School Science \& Technology Fund Planning Project (20140147).

Author Contributions: Jin-Long Yao obtained literatures, classified the chemical constituents and drafted the structural formulas, wrote the manuscript; Shi-Ming Fang classified the chemical constituents and wrote the manuscript; Rui Liu classified the pharmacological literatures and revised the review critically for important intellectual content; Mahmood Brobbey Oppong modified the language; Er-Wei Liu managed references; Guan-Wei Fan contributed to conception and design of the review; Han Zhang obtained funding, overall responsibility.

Conflicts of Interest: The authors declare no conflict of interest.

\section{References}

1. Pei, J.; Chen, S.L. Flora of China; Science Press: Beijing, China, 1982.

2. Chinese Pharmacopoeia Commission. Pharmacopoeia of the People's Republic of China; Beijing Chemical Industry Press: Beijing, China, 2015.

3. Zheng, C.J.; Li, H.Q.; Ren, S.C.; Xu, C.L.; Rahman, K.; Qin, L.P.; Sun, Y.H. Phytochemical and pharmacological profile of Vitex negundo. Phytother. Res. 2015, 29, 633-647. [CrossRef] [PubMed]

4. Li, C.Z.; Su, Y.F.; Jin, X.J. Advances in studies on chemical constituents from plants of Vitex L. and their bioactivities. Chin. Tradit. Herb. Drugs 2005, 36, 930-938.

5. Wu, C.; Zhang, J.; Yin, Z.Q. Chemical constituents from the fruits of Vitex rotundifolia. Pharm. Biotechnol. 2010, 17, 504-507.

6. Wu, J.; Zhou, T.; Zhang, S.W.; Zhang, X.H.; Xuan, L.J. Cytotoxic terpenoids from the fruits of Vitex trifolia L. Planta Med. 2009, 75, 367-370. [CrossRef] [PubMed]

7. Sharma, R.L.; Prabhakar, A.; Dhar, K.L.; Sachar, A. A new iridoid glycoside from Vitex negundo Linn (Verbenacea). Nat. Prod. Res. 2009, 23, 1201-1209. [CrossRef] [PubMed]

8. Iwagawa, T.; Nakahara, A.; Nakatani, M. Iridoids from Vitex cannabifolia. Phytochemistry 1993, 32, $453-454$. [CrossRef]

9. Yamasaki, T.; Kawabata, T.; Masuoka, C.; Kinjo, J.; Ikeda, T.; Nohara, T.; Ono, M. Two new lignan glucosides from the fruit of Vitex cannabifolia. J. Nat. Med. 2008, 62, 47-51. [CrossRef] [PubMed]

10. Suksamrarn, S.; Kumcharoen, S.; Suksamrarn, A. Iridoids of Vitex limonifolia. Planta. Med. 1999, 65. [CrossRef] [PubMed]

11. Kuruüzüm-Uz, A.; Ströch, K.; Demirezer, L.Ö.; Zeeck, A. Glucosides from Vitex agnus-castus. Phytochemistry 2003, 63, 959-964.

12. Kouno, I.; Inoue, M.; Onizuka, Y.; Fujisaki, T.; Kawano, N. Iridoid and phenolic glucoside from Vitex rotundifolia. Phytochemistry 1988, 27, 611-612. [CrossRef] 
13. Sridhar, C.; Subbaraju, G.V.; Venkateswarlu, Y.; Venugopal, R.T. New acylated iridoid glucosides from Vitex altissima. J. Nat. Prod. 2004, 67, 2012-2016. [CrossRef] [PubMed]

14. One, M.; Ito, Y.; Kubo, S.; Nohara, T. Two new iridoids from Viticis trifoliae fructus (Fruit of Vitex rotundifolia L.). Chem. Pharm. Bull. 1997, 45, 1094-1096. [CrossRef]

15. Okuyama, E.; Fujimori, S.; Yamazaki, M.; Deyama, T. Pharmacologically active components of viticis fructus (Vitex rotundifolia). II. The components having analgesic effects. Chem. Pharm. Bull. 1998, 46, 655-662. [CrossRef] [PubMed]

16. Suksamrarn, A.; Kumpun, S.; Kirtikara, K.; Yingyongnarongkul, B.; Suksamrarn, S. Iridoids with anti-inflammatory activity from Vitex peduncularis. Planta Med. 2002, 68, 72-73. [CrossRef] [PubMed]

17. Gu, Q.; Zhang, X.M.; Jiang, Z.Y.; Chen, J.J.; Zhou, J. Chemical constituents from fruits of Vitex trifolia. Chin. Tradit. Herb. Drugs 2007, 38, 656-659.

18. Li, S.H.; Qiu, S.X.; Yao, P.; Sun, H.D.; Fong, H.H.S.; Zhang, H.J. Compounds from the fruits of the popular European medicinal plant Vitex agnus-castus in chemoprevention via NADP(H): Quinone oxidoreductase type 1 induction. Evid. Based Complement. Altern. Med. 2013, 2013, 77-133. [CrossRef] [PubMed]

19. Arai, M.A.; Fujimatsu, T.; Uchida, K.; Sadhu, S.K.; Ahmed, F.; Ishibashi, M. Hh signaling inhibitors from Vitex negundo; naturally occurring inhibitors of the GLI1-DNA complex. Mol. BioSyst. 2013, 9, 1012-1018. [CrossRef] [PubMed]

20. Pandey, A.; Bani, S.; Satti, N.K.; Gupta, B.D.; Suri, K.A. Anti-arthritic activity of agnuside mediated through the down-regulation of inflammatory mediators and cytokines. Inflamm. Res. 2012, 61, 293-304. [CrossRef] [PubMed]

21. Tiwari, N.; Luqman, S.; Masood, N.; Gupta, M.M. Validated high performance thin layer chromatographic method for simultaneous quantification of major iridoids in Vitex trifolia and their antioxidant studies. J. Pharm. Biomed. Anal. 2012, 61, 207-214. [CrossRef] [PubMed]

22. Ramazanov, N.S. Ecdysteroids and iridoidal glycosides from Vitex agnus-castus. Chem. Nat. Compd. 2004, 40, 299-300. [CrossRef]

23. Huang, J.; Wang, G.C.; Wang, C.H.; Huang, X.J.; Ye, W.C. Two new glycosides from Vitex negundo. Nat. Prod. Res. 2013, 27, 1837-1841. [CrossRef] [PubMed]

24. Tasduq, S.A.; Kaiser, P.J.; Gupta, B.D.; Gupta, V.K.; Johri, R.K. Negundoside, an iridiod glycoside from leaves of Vitex negundo, protects human liver cells against calcium-mediated toxicity induced by carbon tetrachloride. World J. Gastroenterol. 2008, 14, 3693-3709. [CrossRef] [PubMed]

25. Manikandan, R.; Thiagarajan, R.; Beulaja, S.; Sivakumar, M.R.; Meiyalagan, V.; Sundaram, R.; Arumugam, M. 1,2 di-substituted idopyranose from Vitex negundo L. protects against streptozotocin-induced diabetes by inhibiting nuclear factor-kappa B and inducible nitric oxide synthase expression. Microsc. Res. Tech. 2011, 74, 301-307. [PubMed]

26. Sundaram, R.; Naresh, R.; Shanthi, P.; Sachdanandam, P. Antihyperglycemic effect of iridoid glucoside, isolated from the leaves of Vitex negundo in streptozotocin-induced diabetic rats with special reference to glycoprotein components. Phytomedicine 2012, 19, 211-216. [CrossRef] [PubMed]

27. Sundaram, R.; Naresh, R.; Ranadevan, R.; Shanthi, P.; Sachdanandam, P. Effect of iridoid glucoside on streptozotocin induced diabetic rats and its role in regulating carbohydrate metabolic enzymes. Eur. J. Pharmacol. 2012, 674, 460-467. [CrossRef] [PubMed]

28. Zheng, C.J.; Pu, J.; Zhang, H.; Han, T.; Rahman, K.; Qin, L.P. Sesquiterpenoids and norterpenoids from Vitex negundo. Fitoterapia 2012, 83, 49-54. [CrossRef] [PubMed]

29. Tiwari, N.; Yadav, A.K.; Vasudev, P.G.; Gupta, M.M. Isolation and structure determination of furanoeremophilanes from Vitex negundo. Tetrahedron. Lett. 2013, 54, 2428-2430. [CrossRef]

30. Barbosa, L.C.A.; Demuner, A.J.; Howarth, O.W.; Pereira, N.S.; Veloso, D.P. Chemical study of the leaves of Vitex poligama. Fitoterapia 1995, LXVI, 279-280.

31. Hajdú, Z.; Hohmann, J.; Forgo, P.; Martinek, T.; Dervarics, M.; Zupkó, I.; Falkay, G.; Cossuta, D.; Máthé, I. Diterpenoids and flavonoids from the fruits of Vitex agnus-castus and antioxidant activity of the fruit extracts and their constituents. Phytother. Res. 2007, 21, 391-394. [CrossRef] [PubMed]

32. Ono, M.; Nagasawa, Y.; Ikeda, T.; Tsuchihashi, R.; Okawa, M.; Kinjo, J.; Yoshimitsu, H.; Nohara, T. Three new diterpenoids from the fruit of Vitex agnus-castus. Chem. Pharm. Bull. 2009, 57, 1132-1135. [CrossRef] [PubMed] 
33. Ono, M.; Eguchi, K.; Konoshita, M.; Furusawa, C.; Sakamoto, J.; Yasuda, S.; Ikeda, T.; Okawa, M.; Kinjo, J.; Yoshimitsu, H.; et al. A new diterpenoid glucoside and two new diterpenoids from the fruit of Vitex agnus-castus. Chem. Pharm. Bull. 2011, 59, 392-396. [CrossRef] [PubMed]

34. Ono, M.; Ito, Y.; Nohara, T. A labdane diterpene glycoside from fruit of Vitex rotundifolia. Phytochemistry 1998, 48, 207-209. [CrossRef]

35. Pal, M.; Li, S.H.; Tewari, S.K.; Sun, H.D. Diterpenoid compounds from Vitex agnus-castus. Chem. Nat. Compd. 2013, 49, 635-638. [CrossRef]

36. Wang, X.Q.; Zhang, T.; Zheng, B.; Xie, W.D.; Shen, T. Labdane-type diterpenoids from the fruits of Vitex rotundifolia. Bull. Korean. Chem. Soc. 2014, 35, 672-674. [CrossRef]

37. Zheng, C.J.; Huang, B.K.; Wang, Y.; Ye, Q.; Han, T.; Zhang, Q.Y.; Zhang, H.; Qin, L.P. Anti-inflammatory diterpenes from the seeds of Vitex negundo. Bioorg. Med. Chem. 2010, 18, 175-181. [CrossRef] [PubMed]

38. Zheng, C.J.; Zhu, J.Y.; Yu, W.; Ma, X.Q.; Rahman, K.; Qin, L.P. Labdane-type diterpenoids from the fruits of Vitex trifolia. J. Nat. Prod. 2013, 76, 287-291. [CrossRef] [PubMed]

39. Ono, M.; Sawamura, H.; Ito, Y.; Mizuki, K.; Nohara, T. Diterpenoids from the fruits of Vitex trifolia. Phytochemistry 2000, 55, 873-877. [CrossRef]

40. Hoberg, E.; Orjala, J.; Meier, B.; Sticher, O. Diterpenoids from the fruits of Vitex agnus-castus. Phytochemistry 1999, 52, 1555-1558. [CrossRef]

41. Jarry, H.; Spengler, B.; Wuttke, W.; Christoffel, V. In vitro assays for bioactivity-guided isolation of endocrine active compounds in Vitex agnus-castus. Maturitas 2006, 55S, S26-S36. [CrossRef]

42. Asaka, Y.; Kamikawa, T.; Kubota, T. Constituents of Vitex rotundifolia L. fil. Chem. Lett. 1973, 937-940. [CrossRef]

43. Huang, D.N.; Qing, S.; Zeng, G.Y.; Wang, Y.J.; Guo, H.; Tan, J.B.; Zhou, Y.J. Lipophilic components from fructus Viticis negundo and their anti-tumor activities. Fitoterapia 2013, 86, 144-148. [CrossRef] [PubMed]

44. Chen, Y.J.; Li, C.M.; Ling, W.W.; Wan, X.C.; Xia, T.; Du, X.F.; Zhang, Z.Z.; Ling, T.J. A rearranged labdane-type diterpenoid and other constituents from Vitex negundo var. cannabifolia. Biochem. Syst. Ecol. 2012, 40, 98-102. [CrossRef]

45. Lee, C.; Lee, J.W.; Jin, Q.H.; Lee, H.J.; Lee, S.J.; Lee, D.; Lee, M.K.; Lee, C.K.; Hong, J.T.; Lee, M.K.; et al. Anti-inflammatory constituents from the fruits of Vitex rotundifolia. Bioorg. Med. Chem. Lett. 2013, 23, 6010-6014. [CrossRef] [PubMed]

46. Kim, Y.A.; Kim, D.S.; Oh, K.; Seo, Y. Isolation of a new labdane-type diterpene from Vitex rotundifolia. Bull. Korean Chem. Soc. 2013, 34, 3840-3842. [CrossRef]

47. Kondo, Y.; Sugiyama, K.; Nozoe, S. Studies on the constituents of Vitex rotundifolia L. fil. Chem. Pharm. Bull. 1986, 34, 4829-4832. [CrossRef] [PubMed]

48. Kiuchi, F.; Matsuo, K.; Ito, M.; Qui, T.K.; Honda, G. New norditerpenoids with trypanocidal activity from Vitex trifolia. Chem. Pharm. Bull. 2004, 52, 1492-1494. [CrossRef] [PubMed]

49. Yan, L.H.; Zhang, Q.W.; Wang, Z.M.; Xu, L.Z.; Yang, S.L. Chemical constituents in Vitex trifolia L. (II). Chin. Tradit. Herb. Drugs 2010, 41, 1622-1624.

50. Ono, M.; Yamamoto, M.; Yanaka, T.; Ito, Y.; Nohara, T. Ten new labdane-type diterpenes from the fruit of Vitex rotundifolia. Chem. Pharm. Bull. 2001, 49, 82-86. [CrossRef] [PubMed]

51. Chen, S.N.; Friesen, J.B.; Webster, D.; Nikolic, D.; van Breemen, R.B.; Wang, Z.J.; Fong, H.H.S.; Farnsworth, N.R.; Pauli, G.F. Phytoconstituents from Vitex agnus-castus fruits. Fitoterapia 2011, 82, 528-533. [CrossRef] [PubMed]

52. Högner, C.; Sturm, S.; Seger, C.; Stuppner, H. Development and validation of a rapid ultra-high performance liquid chromatography diode array detector method for Vitex agnus-castus. J. Chromatogr. B. 2013, 927, 181-190. [CrossRef] [PubMed]

53. Tiwari, N.; Thakur, J.; Saikia, D.; Gupta, M.M. Antitubercular diterpenoids from Vitex trifolia. Phytomedicine 2013, 20, 605-610. [CrossRef] [PubMed]

54. Zheng, C.J.; Huang, B.K.; Wu, Y.B.; Han, T.; Zhang, Q.Y.; Zhang, H.; Qin, L.P. Terpenoids from Vitex negundo seeds. Biochem. Syst. Ecol. 2010, 38, 247-249. [CrossRef]

55. Corlay, N.; Lecsö-Bornet, M.; Leborgne, E.; Blanchard, F.; Cachet, X.; Bignon, J.; Roussi, F.; Butel, M.J.; Awang, K.; Litaudon, M. Antibacterial labdane diterpenoids from Vitex vestita. J. Nat. Prod. 2015, 78, 1348-1356. [CrossRef] [PubMed] 
56. Nyiligira, E.; Viljoen, A.M.; van Heerden, F.R.; van Zyl, R.L.; van Vuuren, S.F.; Steenkamp, P.A. Phytochemistry and in vitro pharmacological activities of South African Vitex (Verbenaceae) species. J. Ethnopharmacol. 2008, 119, 680-685. [CrossRef] [PubMed]

57. Ono, M.; Yamasaki, T.; Konoshita, M.; Ikeda, K.; Okawa, M.; Kinjo, J.; Yoshimitsu, H.; Nohara, T. Five new diterpenoids, viteagnusins A-E, from the fruit of Vitex agnus-castus. Chem. Pharm. Bull. 2008, 56, 1621-1624. [CrossRef] [PubMed]

58. Ono, M.; Yamamoto, M.; Masuoka, C.; Ito, Y.; Yamashita, M.; Nohara, T. Diterpenes from the fruits of Vitex rotundifolia. J. Nat. Prod. 1999, 62, 1532-1537. [CrossRef] [PubMed]

59. Zheng, C.J.; Lan, X.P.; Wang, Y.; Huang, B.K.; Han, T.; Zhang, Q.Y.; Qin, L.P. A new labdane diterpene from Vitex negundo. Pharm. Biol. 2012, 50, 687-690. [CrossRef] [PubMed]

60. Aphaijitt, S.; Nimgirawath, K.; Suksamrarn, A.; Tooptakong, U. Isolation and crystal structure of Limonidilactone-A labdane diterpene from Vitex limonifolia. Aust. J. Chem. 1995, 48, 133-137. [CrossRef]

61. Ono, M.; Yanaka, T.; Yamamoto, M.; Ito, Y.; Nohara, T. New diterpenes and norditerpenes from the fruits of Vitex rotundifolia. J. Nat. Prod. 2002, 65, 537-541. [CrossRef] [PubMed]

62. Zhang, T.; Zhang, C.X.; Xie, W.D.; Row, K.H. Vitrifolin A: A norlabdane diterpenoid from the fruits of Vitex trifolia Linn. var. simplicifolia Cham. J. Chin. Chem. Soc. 2013, 60, 542-545. [CrossRef]

63. Ono, M.; Nishida, Y.; Masuoka, C.; Li, J.C.; Okawa, M.; Ikeda, T.; Nohara, T. Lignan derivatives and a norditerpene from the seeds of Vitex negundo. J. Nat. Prod. 2004, 67, 2073-2075. [CrossRef] [PubMed]

64. Ono, M.; Ito, Y.; Nohara, T. Four new halimane-type diterpenes, vitetrifolins D-G, from the fruit of Vitex trifolia. Chem. Pharm. Bull. 2001, 49, 1220-1222. [CrossRef] [PubMed]

65. Chawla, A.S.; Sharma, A.K.; Handa, S.S.; Dhar, K.L.J. Chemical investigation and anti-inflammatory activity of Vitex negundo seeds, Part I. Indian. J. Chem. 1991, 30B, 773-776.

66. Sakurai, A.; Okamoto, Y.; Kokubo, S.; Chida, A. Abietane-type diterpenoids from the fruit of Vitex rotundifolia L. fil. J. Chem. Soc. Jpn. Chem. Ind. Chem. 1999, 3, 207-211. [CrossRef]

67. Chen, J.; Fan, C.L.; Wang, Y.; Ye, W.C. A new triterpenoid glycoside from Vitex negundo. Chin. J. Nat. Med. 2014, 12, 0218-0221. [CrossRef]

68. Mohamed, M.A.; Abdou, A.M.; Hamed, M.M.; Saad, A.M. Characterization of bioactive phytochemical from the leaves of Vitex trifolia. Int. J. Pharm. Appl. 2012, 3, 419-428.

69. Li, M.M.; Su, X.Q.; Sun, J.; Gu, Y.F.; Huang, Z.; Zeng, K.W.; Zhang, Q.; Zhao, Y.F.; Ferreira, D.; Zjawiony, J.K.; et al. Anti-inflammatory ursane- and oleanane-type triterpenoids from Vitex negundo var. cannabifolia. J. Nat. Prod. 2014, 77, 2248-2254. [CrossRef] [PubMed]

70. Chen, Y.S.; Lin, X.Y.; Zhong, L.J.; Xie, J.M.; Zhang, Y.H. Chemical constituents of Vitex trifolia L. Nat. Prod. Res. Dev. 2011, 23, 1011-1013.

71. Noel, M.G.; Dayrit, F.M. Triterpenes in the callus culture of Vitex negundo L. Philipp. J. Sci. 2005, 134, 5-19.

72. Wang, Y.J.; He, X.; Zeng, G.Y.; Tan, J.B.; Li, X.; Zhou, Y.J. Constituents of triterpenes in the seeds of Vitex negundo L. Cent. South Pharm. 2012, 10, 409-412.

73. Sridhar, C.; Rao, K.V.; Subbaraju, G.V. Flavonoids, triterpenoids and a lignan from Vitex altissima. Phytochemistry 2005, 66, 1707-1712. [CrossRef] [PubMed]

74. Liu, Q.Y.; Chen, Y.S.; Wang, F.; Chen, S.W.; Zhang, Y.H. Chemical of Vitex trifolia. Zhongguo Zhongyao Zazhi. 2014, 39, 2024-2028. [PubMed]

75. Mishra, S.; Pani, S.R.; Rout, K.K.; Nayak, S.K.; Sahoo, S. Bioassay guided fractionation and hepatoprotective activity of oleanolic acid acetate isolated from Vitex negundo Linn. J. Biol. Act. Prod. Nat. 2014, 4, 89-100.

76. Verma, V.K.; Siddiqui, N.U.; Aslam, M. Phytochemical constituents from the bark of Vitex negundo Linn. Int. J. Pharm. Sci. Rev. Res. 2011, 7, 93-95.

77. Chawla, A.S.; Sharma, A.K.; Handa, S.S. Chemical investigation and anti-inflammatory activity of Vitex negundo seeds. J. Nat. Prod. 1992, 55, 163-167. [CrossRef] [PubMed]

78. Chen, Y.S.; Xie, J.M.; Yao, H.; Lin, X.Y.; Zhang, Y.H. Studies on the triterpenoids of Vitex trifolia. J. Chin. Med. Mater. 2010, 33, 908-910.

79. Huang, M.Y.; Zhong, L.J.; Xie, J.M.; Wang, F.; Zhang, Y.H. A new taraxastane-type triterpene from Vitex trifolia var. simplicifolia. Helv. Chim. Acta 2013, 96, 2040-2045. [CrossRef]

80. Sahu, N.P.; Roy, S.K.; Mahato, S.B. Triterpenoids and flavonoids of Vitex peduncularis. Planta Med. 1984, 50, 527. [CrossRef] [PubMed] 
81. Chandramu, C.; Manohar, R.D.; Krupadanam, D.G.L.; Dashavantha, R.V. Isolation, characterization and biological activity of betulinic acid and ursolic acid from Vitex negundo L. Phytother. Res. 2003, 17, 129-134. [CrossRef] [PubMed]

82. Jangwan, J.S.; Aquino, R.P.; Mencherini, T.; Picerno, P.; Singh, R. Chemical constituents of ethanol extract of leaves and molluscicidal activity of crude extracts from Vitex trifolia Linn. Herba Pol. 2013, 59, 19-32. [CrossRef]

83. Rudrapaul, P.; Sarma, I.S.; Das, N.; De, U.C.; Bhattacharjee, S.; Dinda, B. New flavonol methyl ether from the leaves of Vitex peduncularis exhibits potential inhibitory activity against Leishmania donovani through activation of iNOS expression. Eur. J. Med. Chem. 2014, 87, 328-335. [CrossRef] [PubMed]

84. Kannathasan, K.; Senthilkumar, A.; Venkatesalu, V. Crystal structure and antibacterial evaluation of epifriedelinol isolated from Vitex peduncularis Wall. ex Schauer. Arab. J. Chem. 2015. [CrossRef]

85. Arumanyagam, S.; Arunmani, M.; Zariya, J.S.; Geetha, S. Hepatoprotective effect of Vitex negundo Linn. against $\mathrm{CCl}_{4}$. World J. Pharm. Res. 2015, 4, 1313-1325.

(C) 2016 by the authors; licensee MDPI, Basel, Switzerland. This article is an open access article distributed under the terms and conditions of the Creative Commons Attribution (CC-BY) license (http://creativecommons.org/licenses/by/4.0/). 